Draft VERSion July 18, 2016

Preprint typeset using $\mathrm{IAT}_{\mathrm{E}} \mathrm{X}$ style emulateapj v. 5/2/11

\title{
A COMMON ORIGIN FOR GLOBULAR CLUSTERS AND ULTRA-FAINT DWARFS IN SIMULATIONS OF THE FIRST GALAXIES
}

\author{
Massimo Ricotti ${ }^{1}$, Owen H. Parry ${ }^{1}$, Nickolay Y. Gnedin ${ }^{2,3,4}$ \\ Draft version July 18, 2016
}

\begin{abstract}
In this paper, the first in a series on galaxy formation before reionization, we focus on understanding what determines the size and morphology of stellar objects in the first low mass galaxies, using parsecscale cosmological simulations performed with an adaptive mesh hydrodynamics code. Although the dense gas in which stars are formed tends to have a disk structure, stars are found in spheroids with little rotation. Halos with masses between $10^{6} \mathrm{M}_{\odot}$ and $5 \times 10^{8} \mathrm{M}_{\odot}$ form stars stochastically, with stellar masses in the range $10^{4} \mathrm{M}_{\odot}$ to $2 \times 10^{6} \mathrm{M}_{\odot}$. Nearly independent of stellar mass, we observe a large range of half-light radii for the stars, from a few parsecs to a few hundred parsecs and surface brightnesses and mass-to-light ratios ranging from those typical of globular clusters to ultra-faint dwarfs.

In our simulations, stars form in dense stellar clusters with high gas-to-star conversion efficiencies and rather uniform metallicities. A fraction of these clusters remain bound after the gas is removed by feedback, but others are destroyed, and their stars, which typically have velocity dispersions of 20 to $40 \mathrm{~km} \mathrm{~s}^{-1}$, expand until they become bound by the dark matter halo. We thus speculate that the stars in ultra-faint dwarf galaxies may show kinematic and chemical signatures consistent with their origin in a few distinct stellar clusters. On the other hand, some globular clusters may form at the center of primordial dwarf galaxies and may contain dark matter, perhaps detectable in the outer parts.
\end{abstract}

Subject headings: cosmology: theory - methods: numerical - galaxies: formation - galaxies: evolution

\section{INTRODUCTION}

The epoch of formation of the first stars and galaxies is poorly known due to lack of direct observations and the difficulty of ab-initio theoretical modeling. However, understanding this short cosmic epoch is of great importance, not only on its own merit, but also to make progress in other fields of research in astrophysics such as near-field cosmology (e.g., Ricotti \& Gnedin 2005; Bovill \& Ricotti 2011b: Wheeler et al. 2015), the epoch of reionization (e.g., Gnedin 2014: Wise et al. 2014: O'Shea et al. 2015 and the formation of the progenitors of supermassive black holes (e.g., Volonteri 2010 Katz et al. 2015).

It has been established that the first stars in the universe (Population III) have unique properties because they formed in gas of primordial composition, thus devoid of important coolants such as carbon and oxygen, and with inefficient $\mathrm{H}_{2}$ formation because of the absence of dust. Simulations of the formation of Population III stars (Bromm et al. 1999; Abel et al. 2000 Turk et al. 2009) have shown that gas condenses at the center of minihalos of mass $10^{5}-10^{6} \mathrm{M}_{\odot}$, reaching densities of the order of $10^{11} \mathrm{~cm}^{-3}$ on 100 AU scales. Initially the density of the gas increases, driven by the gravitational potential of the dark matter. As the gas becomes fully molec-

\footnotetext{
${ }^{1}$ Department of Astronomy, University of Maryland, College Park, MD 20740, USA; ricotti@astro.umd.edu, oparry@umd.edu

2 Particle Astrophysics Center, Fermi National Accelerator Laboratory, Batavia, IL 60510, USA

${ }^{3}$ Kavli Institute for Cosmological Physics and Enrico Fermi Institute, The University of Chicago, Chicago, IL 60637 USA

${ }^{4}$ Department of Astronomy \& Astrophysics, University of Chicago, Chicago, IL 60637 USA
}

ular, the gas cools further and becomes self-gravitating forming a proto-star (Abel et al. 2002). In order to numerically resolve the starting phases of star formation in metal-free and dust-free molecular clouds it is therefore necessary to achieve a numerical resolution of a few $100 \mathrm{AU}$ (or $1 \mathrm{M}_{\odot}$ mass scale). As the first stars synthesize and eject metals into the intergalactic medium (IGM) and interstellar medium (ISM), the metallicity and dust-to-gas ratio in star forming regions increase and the enhanced cooling rates allow molecular clouds to form at lower mean density, and on larger mass and spatial scales. Thus, the numerical requirements for resolving star formation in molecular clouds become less stringent with increasing gas metallicity (e.g., Bromm et al. 2001: Kuhlen et al. 2013: Tomassetti et al. 2015).

Two approaches are widely used for modeling the formation of the first dwarf galaxies in cosmological simulations. In the first approach metal enrichment is calculated self-consistently resolving the formation of the first stars at $z>10$ in relatively small (1-4 comoving $\mathrm{Mpc}^{3}$ ) cosmological volumes (Ricotti et al. 2002a b: Wise \& Abel 2007; Ricotti et al. 2008a; Muratov et al. 2013a b Wise et al. 2014). The second approach, typically used in "zoom simulations" of dwarf galaxies, adopts a metallicity floor (typically $Z \sim 10^{-3} \mathrm{Z}_{\odot}$ ) everywhere in the IGM in order to initiate normal Population II star formation avoiding to capture Population III star formation (Gnedin et al. 2009, Tassis et al. 2012, Christensen et al. 2012; Kuhlen et al. 2013; Hopkins et al. 2014; Thompson et al. 2014; Wheeler et al. 2015)

It is not difficult to suppress Population II star formation in dwarf halos. First, photo-heating during reion- 
ization shuts off accretion below a characteristic mass $M_{c}(z)$, and internal ionization sources can unbind the majority of baryons altogether (Efstathiou 1992; Barkana \& Loeb 1999; Gnedin 2000; Hoeft et al. 2006; Okamoto et al. |2008; Simpson et al. |2013, Benitez-Llambay et al. 2014 Ricotti 2009 Pawlik et al. 2009: Sawala et al. 2014). Second, slow formation of $\mathrm{H}_{2}$ in pristine almost-dust-free gas can also inhibit star formation. Simulations that model molecular chemistry and form stars in molecular clouds confirm that dwarf halos can be left almost totally dark because gas fails to collapse to sufficient density for dust to form, and cooling to continue (Gnedin et al. 2010; Kuhlen et al. 2012, 2013, Jaacks et al. |2013, Thompson et al. 2014). However, not surprisingly in light of the discussion above, a number of authors have found that results for low-metallicity dwarf galaxies depend on the chosen numerical resolution when star formation is restricted to resolution elements in which the gas is fully molecular (Hopkins et al. 2012, Wise et al. 2014, Kimm \& Cen 2014; Tomassetti et al. 2015). Thus, adopting such a sub-grid recipe in simulations focused on the transition between Population III to Population II stars may lead to an artificial suppression of star formation in gas with metallicity below a critical threshold set by the numerical resolution.

In this paper, we present new radiation-hydrodynamic cosmological simulations of the formation of the first stars and dwarf galaxies in which we adopt a model for Population III and Population II star formation (i.e., without a metallicity floor) and their radiative and mechanical feedback in small volume simulations (about 1 comoving $\mathrm{Mpc}^{3}$ ). We use the adaptive refinement tree (ART) code with appropriate modifications as described in $\S 2$ and in more detail in a companion paper (Parry, Ricotti \& Gnedin, in preparation). While we cannot resolve all stages of star formation, we do resolve dense clumps of gas on $0.1-1$ pc scales that (if Jeans unstable) inevitably collapse into Population III or Population II stars. Contrary to other implementations of Population II star formation, we do not require the gas to be fully molecular but we set a very high density threshold for star formation by requiring the gas to be self-gravitating and converging at the maximum refinement level (see $\S 2$. With this choice we avoid the metallicity-dependent resolution requirements for the gas to become fully molecular and treat consistently the transition from metal-free to metal-poor star formation. Star formation only takes place on the maximum refinement level on parsec or subparsec scales with efficiency $\epsilon_{*}$ of conversion of $\rho_{\text {gas }}$ into $\rho_{*}$ on a local dynamical timescale (we explore a range for $\epsilon_{*}$ between $1 \%$ and $100 \%$ ). We find that at sub-parsec resolution the majority of Population II stars (defined to have metallicity $Z>10^{-5} \mathrm{Z}_{\odot}$ ) form in gas that is $10 \%$ to $60 \%$ molecular, while metal-free gas forming Population III stars is only partially molecular at these scales.

The transition from Population III to Population II star formation has been the focus of previous semianalytical (e.g., Scannapieco et al. 2003, Yoshida et al. 2004 Schneider et al. 2006) and numerical simulations (e.g., Tornatore et al. 2007; Maio et al. 2010, Greif et al. 2011; Johnson et al. 2013; Wise et al. 2012b a; Muratov et al. 2013a b; Wise et al. 2014). This transition is important for predicting whether the James Webb Space
Telescope (JWST) will be able to observe galaxies dominated by Population III stars (Pawlik et al. 2011; Zackrisson et al. 2011), understanding the sources of IGM reionization (Ciardi et al. 2000; Ricotti et al. 2002b; Ricotti \& Ostriker 2004; Gnedin 2008; Ricotti et al. 2008b; Boylan-Kolchin et al. 2014), determining the origin of ultra-faint dwarf (UFD) galaxies in the Local Group and probing dark matter and gravity on small scales (Polisensky \& Ricotti 2011).

Galaxies with masses below a critical value $M_{c}(z)$ are expected to have an early truncation of their star formation histories due to reionization or internal feedback mechanisms, and thus can be used as a laboratory to investigate the conditions and star formation in the high redshift universe Bovill \& Ricotti (2011b); BoylanKolchin et al. (2015). These systems are commonly referred to as "fossil galaxies" (Ricotti \& Gnedin 2005, Bovill \& Ricotti 2009), reflecting the idea that their stellar populations are $>11-12$ Gyr old. The best candidates for such systems are UFDs $\left(\mathrm{L}<10^{5} \mathrm{~L}_{\odot}\right)$ discovered in the past decade (Belokurov et al. 2007, 2010; Koposov et al. 2015; Drlica-Wagner et al. 2015) and some old globular clusters (GCs) (Ricotti 2002, Katz \& Ricotti 2013, 2014). Most UFDs contain only old, metal-poor stars and appear to have simple star formation histories (Brown et al. 2012, 2014), making them excellent candidates for probing chemical and dynamical signatures of the first generations of stars.

The focus of this paper is on how morphology begins to develop in the earliest galaxies, before the epoch of reionization, as well the relationship between morphology and the kinematic properties of the stars and gas, their metallicities and modes of star formation. In particular, we are interested in establishing observational links between simulated fossils galaxies and dwarf spheroidals and UFDs in the Local Group for which detailed properties (including morphology, kinematics and chemistry) are available, or can be probed by targeted observations. In previous works (Ricotti \& Gnedin 2005, Bovill \& Ricotti 2009), we found that the simulated fossils have stellar spheroids with half-light radii of about 100 pc (nearly independent of their luminosity and mass-to-light ratio) and surface brightnesses consistent with the faintest dwarf spheroidals and UFDs. Here, we re-examine this question using simulations that have much higher spatial resolution (about a factor of ten higher) and that, by allowing star formation on sub-parsec scales, can resolve the eventual formation of compact star clusters. These simulations are similar to the ones presented in Muratov et al. (2013a), as they are run with ART and adopt similar (but not identical) sub-grid recipes for star formation and feedback. Another difference in our simulation with respect to previous works (e.g., Wise et al. 2012c 2014 ) is that we use a star formation recipe and radiation transfer methods (OTVET) that allows the formation of hundreds to several tens of thousands of "star particles" per galaxy, with masses as low as $\sim 40 \mathrm{M}_{\odot}$. We also form stars stochastically, checking for suppression of star formation due to feedback with time resolution of $10^{5}$ years. The masses of the stellar particles are not small enough for sampling a realistic IMF, but they are much smaller than a cluster mass and allow us to resolve the formation of compact star clusters that may remain bound if their star formation efficiency is close to $50 \%$ 
(Hills 1980; Geyer \& Burkert 2001). This is of crucial importance because in the local universe star formation is observed to occur only in clusters (Lada \& Lada 2003) and likely the same is true at high redshift (Clark et al. 2008 Karlsson et al. 2012). However previous cosmological simulations, (especially the ones that use ray-tracing for radiative transfer) could not afford to form individual stars, so instead employed stellar particles representing whole clusters of stars.

The numerical improvement described above allows us to revisit important questions on the morphology an chemical signatures in the first galaxies:

- "Emergence of the Hubble sequence": Is there a genuine trend for more irregular and spheroidal galaxies at high-z or vice versa gas-rich disks are common?

- "Origin of dSphs vs dIrrs": Are the morphologies of dSphs and UFDs in the local group set at formation or are the result of tidal interactions with the Milky-Way?

- "Size and surface brightness of fossil galaxies": What determines the half-light radii and surface brightnesses of dSphs and UFDs?

- "Unified theoretical model for the formation of compact stellar clusters and UFDs": The discovery of UFDs and dwarf-globular transition objects (e.g., Willman \& Strader 2012; Forbes et al. 2013) somewhat blurred the distinction between compact stellar clusters and dwarf galaxies. Is there a deeper link between these objects?

The layout of this paper is as follows. In Section 2 we briefly describe the cosmological code ART used in this work, initial conditions and the physics included in the simulation, as well as the modifications we have made to model metal-free star formation. Our results are presented in Section 3 and a discussion in Section 4. Summary and conclusions are in Section 5 .

\section{THE SIMULATIONS}

Our simulations follow the evolution of an ensemble of galaxies in a $1 \mathrm{~h}^{-1} \mathrm{Mpc}$ volume of the Universe prior to the epoch of reionization. In practice, the end point of the simulations is $z \sim 9$. The simulation code ART (Adaptive Refinement Tree; Kravtsov et al. 1997; Kravtsov 1999,2003 , Rudd et al. 2008), employs an Eulerian scheme to track the dynamics of gas, dark matter and stars, adaptively improving the spatial and temporal resolution in the densest and most rapidly evolving regions of the simulation volume. The propagation of ionizing photons emitted by young stars is tracked through radiative transfer calculations that are self-consistently coupled to the hydrodynamics. The code also includes a prescription for modeling the abundance of molecular Hydrogen $\left(\mathrm{H}_{2}\right)$ that includes $\mathrm{H}^{-}$catalyzed formation and formation on dust grains, self-shielding and dust shielding (Gnedin et al. 2009, Gnedin \& Kravtsov 2011). Full details of our version of ART can be found in Section 2 of a companion paper (Parry, Ricotti, Gnedin 2016, in preparation) (hereafter PRG16) and references therein. The following subsections recap those parts of the code that are particularly relevant to dynamics and morphology in the first galaxies.

Three-dimensional radiative transfer in four energy bands is solved and coupled to the hydrodynamics calculation using the the OTVET approximation (Gnedin \& Abel 2001). We consider the propagation of $\mathrm{H} \mathrm{I}$, $\mathrm{He} \mathrm{I}$ and $\mathrm{He} \mathrm{II}$ ionizing photons, as well as $\mathrm{H}_{2}$ dissociating photons in the Lyman-Werner bands. Self-shielding and dust-shielding of $\mathrm{H}_{2}$ are included using an observationally motivated model. Both shielding factors are computed as functions of the local column densities of $\mathrm{H}_{\mathrm{I}}$ and $\mathrm{H}_{2}$, which are approximated as $n \rho /|\nabla \rho|$. ART keeps track of a non-equilibrium chemical network that includes five species of atomic and ionized Hydrogen and Helium, as well as molecular Hydrogen. The abundances of each species, together with the local UV radiation intensity, are used to compute self-consistent heating and cooling rates.

\subsection{Initial Conditions}

The simulations presented here begin from two different sets of initial conditions, both of which have formed part of previously published works; Ricotti et al. (2002a b) (hereafter R02) and Muratov et al. (2013a b) (hereafter AM13). Both assume a $\Lambda$ CDM cosmology and represent a cubic volume of the Universe, $1 \mathrm{~h}^{-1} \mathrm{Mpc}$ on a side. The properties of the two sets of initial conditions are listed in Table 1

In all of our simulations the number of dark matter particles, $N_{\mathrm{DM}}$, is equal to the number of cells in the root level mesh $N_{\text {root }}$, which sets the particle mass, $M_{\mathrm{DM}}=\rho_{\text {crit }} \Omega_{D M} V_{\text {sim }} / N_{\text {root }}$, where $\rho_{\text {crit }}$ is the critical density and $V_{\text {sim }}$ is the simulation volume.

\subsection{Mesh Refinement}

Three criteria control the refinement of the simulation mesh. Cells are refined when their gas or dark matter masses exceed the threshold values $M_{\text {gas,th }}$ and $M_{\mathrm{DM}, \mathrm{th}}$. We set $M_{\mathrm{DM} \text {,th }}$ equal to the mass of one dark matter particle and the gas mass threshold is then fixed at $M_{\mathrm{DM} \text {,th }} \times \Omega_{b} / \Omega_{\mathrm{DM}}$, such that the two refinement criteria are equivalent for a cell with the cosmic baryon fraction. In addition, we require that the Jeans length of the gas, $L_{\mathrm{J}}=20.7 \mathrm{pc}(T / n)^{1 / 2}$, be resolved by at least 5 cell lengths, which satisfies the condition described by Truelove et al. (1997) required to avoid artificial fragmentation.

\subsection{Pop II Star Formation and Feedback}

Pop II star formation is allowed only when

1. The cell is maximally refined: $\Delta x=10$ comoving pc (see Table 1).

2. The flow is convergent $(\nabla \cdot \vec{v}<0)$.

3. The Jeans's length of the gas can no longer be resolved by five cells $\left(L_{\mathrm{J}}<5 \Delta x\right)$. For the value of $\Delta x$ in our simulations $L_{\mathrm{J}}<50$ comoving pc, translates in the density threshold for star formation:

$n_{\mathrm{H}}>n_{\mathrm{H}, \mathrm{pII}} \approx 1.7 \times 10^{3} \mathrm{~cm}^{-3}\left(\frac{T}{100 K}\right)\left(\frac{1+z}{10}\right)^{2}$. 
Table 1

The numerical parameters adopted for each of our simulations.

\begin{tabular}{|c|c|c|c|c|c|c|c|c|c|c|c|c|}
\hline Label & $\begin{array}{c}\Delta x \\
\text { com. pc }\end{array}$ & $\begin{array}{l}\mathrm{M}_{\mathrm{DM}, \text { part }} \\
{\left[\begin{array}{ll}10^{3} & \mathrm{M}_{\odot}\end{array}\right]}\end{array}$ & $\mathrm{N}_{\text {levs }}$ & $\begin{array}{c}\mathrm{M}_{*, \min } \\
{\left[\mathrm{M}_{\odot}\right]}\end{array}$ & $\begin{array}{l}Z_{\text {crit }} \\
{\left[\mathrm{Z}_{\odot}\right]}\end{array}$ & $\begin{array}{l}\mathrm{n}_{\mathrm{H}, \mathrm{pIII}} \\
{\left[\mathrm{cm}^{-3}\right]}\end{array}$ & $\begin{array}{c}\mathrm{f}_{\mathrm{H}_{2}, \mathrm{pIII}} \\
{\left[10^{-5}\right]}\end{array}$ & $\begin{array}{c}\mathrm{E}_{\mathrm{SN}, \mathrm{pIII}} \\
{\left[10^{51} \mathrm{ergs}\right]}\end{array}$ & $\begin{array}{c}\mathrm{E}_{\mathrm{SN}, \mathrm{pII}} \\
{\left[10^{51} \mathrm{ergs}\right]}\end{array}$ & $\begin{array}{c}\text { IMF } \\
\text { (Pop II) }\end{array}$ & $\begin{array}{c}t_{*, \mathrm{samp}} \\
{[\mathrm{Myr}]}\end{array}$ & $\epsilon_{*}$ \\
\hline $\mathrm{REF}^{\mathrm{a}}$ & 10.9 & 49.2 & 10 & 40 & $10^{-5}$ & 1.0 & 1.0 & 30 & 1.0 & Chab. & 0.1 & 0.1 \\
\hline HSFE $^{\mathrm{a}}$ & 10.9 & 49.2 & 10 & 40 & $10^{-5}$ & 1.0 & 1.0 & 30 & 1.0 & Chab. & 0.1 & 1.0 \\
\hline $\mathrm{LSFE}^{\mathrm{a}}$ & 10.9 & 49.2 & 10 & 40 & $10^{-5}$ & 1.0 & 1.0 & 30 & 1.0 & Chab. & 0.1 & 0.01 \\
\hline AM13-REF ${ }^{\mathrm{b}}$ & 10.9 & 5.5 & 9 & 40 & $10^{-5}$ & 1.0 & 1.0 & 30 & 1.0 & Chab. & 0.1 & 0.1 \\
\hline
\end{tabular}

a IC with cosmological parameters: $\left(\Omega_{m}, \Omega_{\Lambda}, \Omega_{b}, \sigma_{8}, n_{s}\right)=(0.30,0.70,0.040,0.90,1.00)$

b IC with cosmological parameters: $\left(\Omega_{m}, \Omega_{\Lambda}, \Omega_{b}, \sigma_{8}, n_{s}\right)=(0.28,0.72,0.046,0.817,0.96)$

Phase diagrams show that the bulk of Population II star formation takes place at densities $10^{4}-10^{5} \mathrm{~cm}^{-3}$, with maximum gas densities as high as $10^{6} \mathrm{~cm}^{-3}$ in few cells. The star forming cells have temperatures ranging $T \sim 10-10^{3} \mathrm{~K}$ and $f_{H_{2}} \sim 10 \%-60 \%$.

4. We also require that the gas overdensity is at least $\delta_{\text {gas }}>2000$ and the gas temperature $T<10^{4} \mathrm{~K}$.

If all four conditions are met, star formation starts after one dynamical time with rate:

$$
\frac{d \rho_{*}}{d t}=\epsilon_{*} \frac{\rho_{\mathrm{gas}}}{t_{\mathrm{dyn}}}
$$

where $\rho_{\text {gas }}$ is the gas density, $t_{\text {dyn }}=\left(3 \pi / 32 G \rho_{\text {gas }}\right)^{0.5}$ is the dynamical time and $\epsilon_{*}$ is the fraction of gas converted into stars in $t_{\mathrm{dyn}}$. Note that - similarly to the recipe for Population III star formation discussed below - we do not require the gas to be fully molecular $\left(f_{H_{2}}=100 \%\right)$.

Each Pop II star particle represents a stellar population with a Chabrier (2003) initial mass function (IMF). They can lose mass through stellar winds associated with massive stars and through the ejection of material by supernovae (SNe). Stellar particles emit radiation with the spectral energy distribution (SED) shown in figure 4 of Ricotti et al. (2002a) and an overall normalization that evolves with age according to the Starburst99 model of Leitherer et al. (1999). This results in a radiative output that begins to fall off rapidly after 3 Myr and has dropped by four orders of magnitude after $\sim 30$ Myr. Stars are formed stochastically over a typical time scale of 1 Myr and have a minimum mass of $\mathrm{M}_{*, \min }=40 \mathrm{M}_{\odot}$. Conditions for star formation are checked every $t_{* \text {,samp }}=0.1$ Myr. SNe explosions begin after a time delay equal to the lifetime of a $8 \mathrm{M}_{\odot}$ star (3.4 Myr) and continue for a total of $35 \mathrm{Myr}$, with each SN generating $E_{\mathrm{SN}, \mathrm{pII}}=10^{51}$ ergs of thermal energy and ejecting overall a mass in metals equal to $1.1 \%$ of the initial stellar mass. At each time step over this interval, the total energy and metals produced by the stellar population are deposited in the host cell.

\subsection{Pop III Star Formation and Feedback}

To be eligible for Pop III star formation, cells with metallicity less than $Z_{\text {crit }}=10^{-5} \mathrm{Z}_{\odot}$ must have gas densities and molecular fractions exceeding threshold values:

$$
\begin{aligned}
& \text { 1. } n_{\mathrm{H}}>n_{\mathrm{H}, \mathrm{pIII}}=1.0 \mathrm{~cm}^{-3} \text {, } \\
& \text { 2. } f_{\mathrm{H}_{2}}>f_{\mathrm{H}_{2}, \mathrm{pIII}}=10^{-5} .
\end{aligned}
$$

When these conditions are satisfied, a particle representing a single Pop III star with mass $M_{\mathrm{pIII}}=40 \mathrm{M}_{\odot}$ is formed during the next time step. Each Pop III star has a lifetime of 3.9 Myr and radiates with the same SED used for Pop II star particles, but with an increased ionizing luminosity, in agreement with the model from Schaerer (2002) for a zero metallicity star of $40 \mathrm{M}_{\odot}$. At the end of their lives, Pop III particles explode as hypernovae (Umeda \& Nomoto 2003) and eject $8 \mathrm{M}_{\odot}$ of metals and $E_{\mathrm{SN}, \mathrm{pIII}}=30 \times 10^{51} \mathrm{erg}$ of thermal energy into their immediate surroundings. Following Muratov et al. (2013a b), we distribute metal and energy evenly over a sphere of radius 1.5 cell lengths around the star. The assumed thresholds for star formation are also listed in Table 1

\subsection{Analysis}

Unless otherwise specified the results shown in the next sections refer to galaxies identified at redshift $z=9$. We analyze the simulations using two forms of data - log files that record global properties of the simulation at every root-level time step, and output snapshots containing the properties of the dark matter, stars, gas and radiation field throughout the simulation volume. Snapshots are written at values of the expansion factor separated by 0.01 until 0.05 , and by 0.005 thereafter.

Individual galaxies are identified using a modified version of the SUBFIND halo finder code (Springel et al. 2001). The first phase of the algorithm identifies "friends-of-friends" groups (Press \& Davis 1982, Davis et al. 1985) by linking dark matter and star particles separated by less than 0.2 times the mean inter-particle separation. It then finds gravitationally bound substructures (sub-halos) by iteratively unbinding particles around local density peaks. The center of each galaxy is deemed to be the potential minimum within the sub-halo. Although SUBFIND identifies all substructures with at least 32 particles, we impose a more conservative limit of 50, consistent with Kravtsov et al. (2004) who found that the cumulative mass function was converged above that resolution threshold. The minimum galaxy mass considered in the following sections is therefore $2.5 \times 10^{6} \mathrm{M}_{\odot}$ and $2.8 \times 10^{5} \mathrm{M}_{\odot}$ for the REF and AM13-REF simulations respectively. We stress that, while the halo mass function can be considered substantially complete down to this limit, the star formation histories of halos close to the limit will likely be affected by a lack of resolution in their progenitors. Where merger trees are required for the analysis, they are constructed by linking sub-halos in each snapshot with any progenitor in the previous snapshot that contains at least five per cent of their dark 
matter particles.

\section{RESULTS}

\subsection{Gas Disks and Stellar Spheroids}

Fig. 1 shows that gas disks, although rather thick, are clearly identifiable in many of the brightest (top five ranked by stellar mass) galaxies at redshift $=9$. The projected gas density is shown in two orientations for each galaxy, parallel and normal to the angular momentum vector of the gas. In the cases where a well-defined gas disk is present, this gives face-on and edge-on views.

In Fig. 2 we show the projected stellar mass density for the same five galaxies that appear in Fig. 1. The mass from each star particle is spread out over nearby pixels using an SPH-like kerne $!^{5}$ enclosing 32 neighbors. The projection axes and scales of the images are the same as in Fig. 1. The morphology of the stellar component of these galaxies is clearly much closer to a spheroid than that of the gas, although some flattening is apparent in the same sense.

\subsection{Circularity of Stellar Orbits}

A straightforward way to identify stellar disks in simulated galaxies is to compute the circularity $(\mathcal{E})$ of star particle orbits (e.g. Abadi et al. 2003; Scannapieco et al. 2012). In a coordinate system where the net angular momentum of all of the galaxy's stars is in the positive $\mathrm{z}$ direction, circularity may be defined as:

$$
\mathcal{E}_{\mathrm{E}}=\frac{J_{z}}{J_{\text {circ }}(E)},
$$

where $J_{z}$ is the $z$ component of the star's specific angular momentum and $J_{\text {circ }}(E)$ is the specific angular momentum of a star with the same binding energy on a circular orbit. For an infinitely thin, rotationally supported disk, the distribution of circularities is a $\delta$ function at $\mathcal{E}_{\mathrm{E}}=1$, while a non-rotating, dispersion dominated spheroid gives rise to a broad, symmetric distribution peaking at $\mathcal{E}_{\mathrm{E}}=0$.

Fig. 3 shows histograms of $\mathcal{E}_{\mathrm{E}}$ for all star particles in the six brightest galaxies in our REF simulation. Most of the six distributions are consistent with non-rotating spheroids, but in two cases the mean of the distribution is positive, suggesting that the spheroid is rotating, or that a thickened disk structure is superimposed on the non-rotating spheroid. No obvious difference is apparent between metal-rich stars $([\mathrm{Fe} / \mathrm{H}]>-1.5$, red $)$ and metalpoor stars $([\mathrm{Fe} / \mathrm{H}]<-1.5$, blue $)$.

\subsection{Size and Surface Brightness of Stellar Spheroids}

Fig. 4 shows the projected stellar half-mass radii $\left(r_{\mathrm{h}}\right)$ for all galaxies in our REF simulation with five or more star particles. To quantify the effect of different viewing angles, $r_{\mathrm{h}}$ was computed 100 times for each galaxy using random orientations. Error bars indicate the $10^{\text {th }}$ and $90^{\text {th }}$ percentiles of the resulting distribution for each galaxy. In contrast to Local Group dwarfs, which show

\footnotetext{
${ }^{5}$ Letting $\mathrm{u}=\mathrm{r} / \mathrm{h}$, where $\mathrm{r}$ is the $(2 \mathrm{D})$ displacement from the kernel center and $\mathrm{h}$ is the smoothing length, the kernel has the form $w(u)=w_{0}+w_{1}(u-1) u^{2}$ for $u<0.5$ and $w(u)=w_{3}\left(1-u^{3}\right)$ for $u \geq 0.5$. The constants $w_{0}, w_{1}$ and $w_{2}$ are chosen such that $\int_{0}^{1} w(u) \mathrm{d} u=1$.
}

a relatively clear correlation between $r_{\mathrm{h}}$ and stellar mass (e.g. Martin et al. 2008), the simulated galaxies can vary by a factor of 100 in $r_{\mathrm{h}}$ at a fixed stellar mass. The radius $r_{\mathrm{h}}$ is almost independent of viewing angle in many of the galaxies, suggesting relatively spherical distributions, although, as expected, the variation increases at lower $M_{*}$ where there are fewer star particles per galaxy. Note that the points clustered at the top left corner of the figure are not a numerical artifact but halos that only contain Pop III stars (colored in blue). These halos have an extended and low surface brightness stellar spheroid produced by mergers of several minihalos containing Pop III stars. However, if Pop III stars are indeed massive as we have assumed here, the present-day fossil remnants of these objects would be totally dark.

In order to make sure that the spatial distribution and kinematics of the stars are not affected by numerical issues, we performed a series of tests. First, we estimated the stellar radii of the spheroids by defining a radius containing half of the star particles, rather than half of the stellar mass. Given that star particles in our simulations have a wide range of masses, we checked whether a few massive star particles that sank to the center of the halo could be biasing the half-mass radii to smaller values. Indeed, it makes sense that star particles, which represent a collection of stars, should be treated as an extended distribution, rather than a point mass. We found no significant differences between the half-mass radius and the radius containing half of the star particles in each case. Thus, the compact star clusters with half-mass radii of a few pc in our simulations are not a numerical artifact, but truly a compact collection of many star particles in virial equilibrium (see below).

The second test regards the kinematics of the stars. We wanted to check whether the half-mass radii are different for lighter star particles than for heavier ones. This would be the case if unphysical 2-body interactions between light star particles and dark matter or massive stellar particles were to dynamically heat the system. We found that, excluding progressively more massive subsets of particles, starting with the lightest, there was no obvious effect on the calculated value of $r_{\mathrm{h}}$.

\subsection{Properties of Compact Stellar Clusters and Low Surface Brightness Dwarfs}

Fig. 5(left panel) shows the dark matter mass as a function of the stellar half-mass radius for all galaxies with more than five star particles in the REF simulation. The color coding shows bound objects that have only Pop II stars (red), only Pop III (blue) and both (black). In this plot we note a few interesting properties:

- Normal dwarf galaxies: These are bound objects with $M_{d m} \geq 10^{8} \mathrm{M}_{\odot}$ and contain both Pop II and Pop III stars.

- Failed dwarf galaxies or "dark galaxies": These are bound objects with $M_{d m} \sim 10^{7} \mathrm{M}_{\odot}$ that failed to form Pop II stars (about 13 objects). They contain only Pop III stars distributed in a relatively extended stellar halo ( $>50 \mathrm{pc})$. Their fossils today would be dark, unless low mass Pop III exist.

- Triggered star formation and compact stellar clusters: These are bound objects with $M_{d m} \leq 10^{6} \mathrm{M}_{\odot}$ 


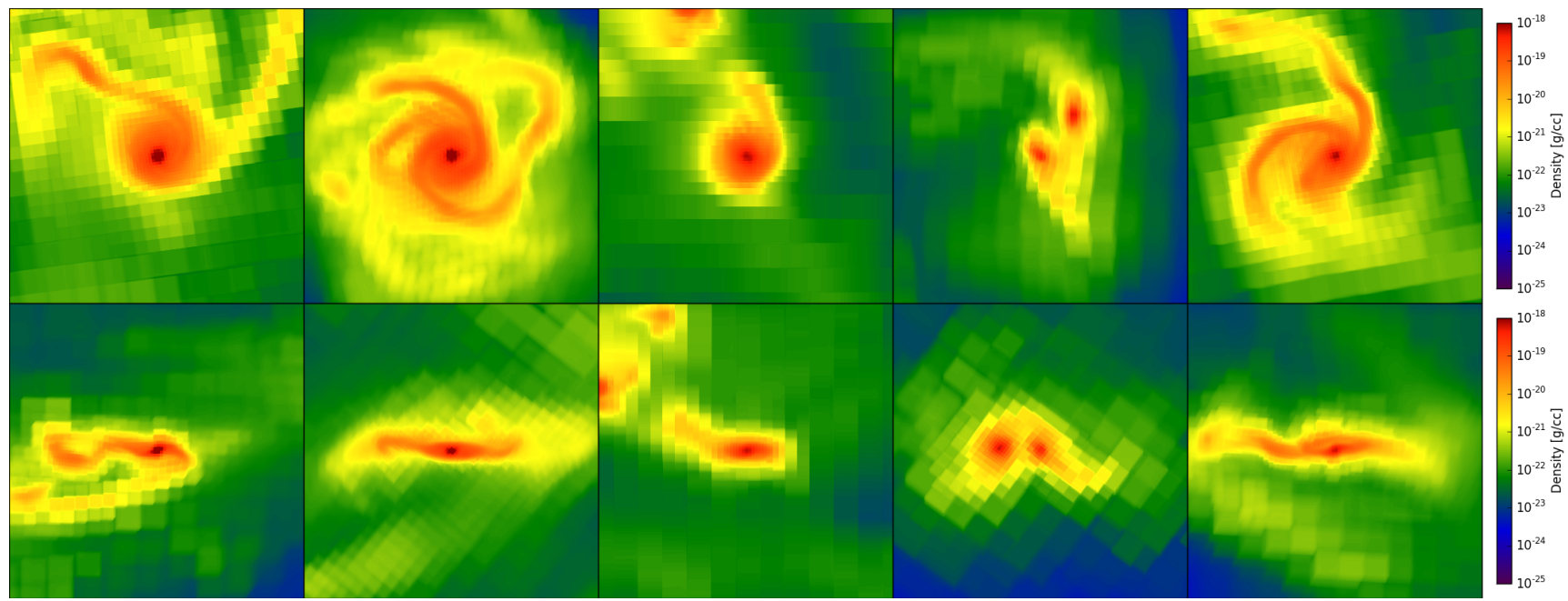

Figure 1. Projected gas density in the top five galaxies ranked by stellar mass at $z=9$. In the top row the projection direction is parallel to the net angular momentum vector of all gas within 2 per cent of the virial radius; in the bottom row, the projection direction is normal to that vector. Each image is $100 \mathrm{pc}$ on a side. Image produced using the yt python libraries, (Turk et al. 2011).

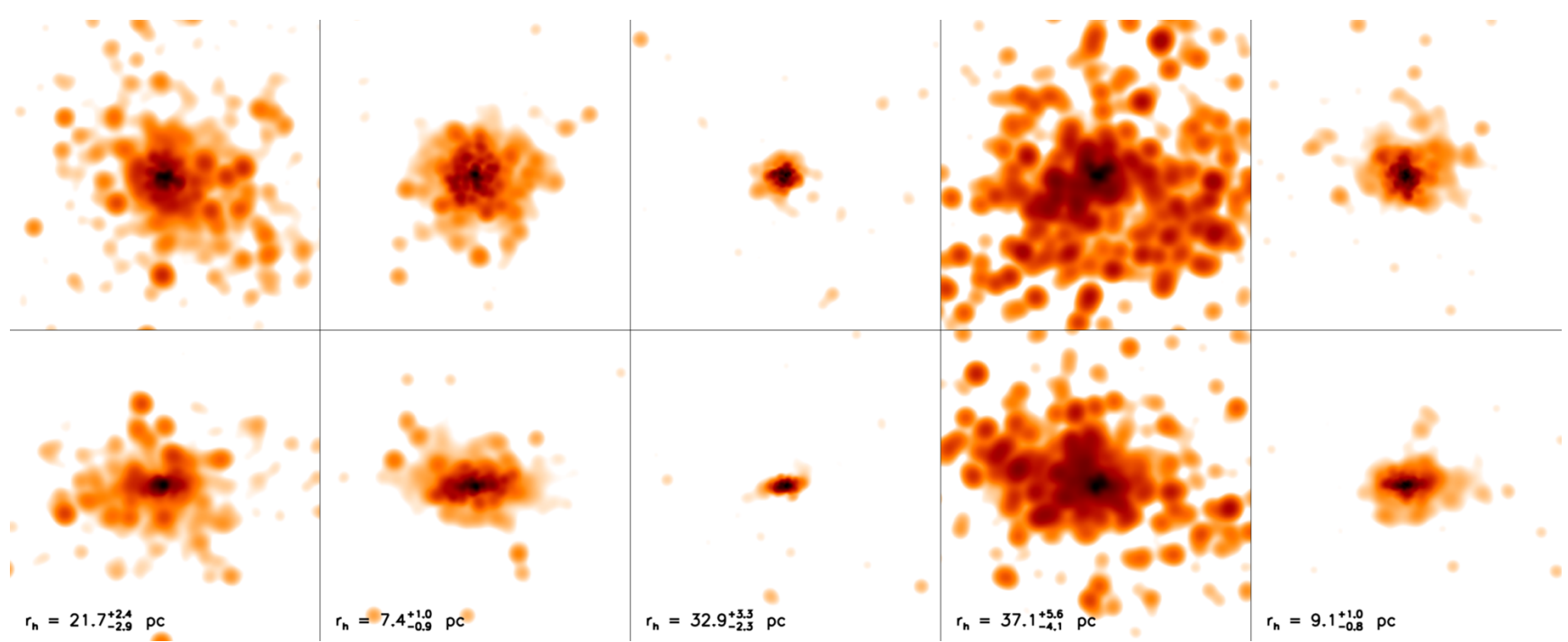

Figure 2. Images of the smoothed projected stellar density in the top five galaxies ranked by stellar mass (counterparts to the images in Fig. 1) at $z=9$. Details of the smoothing technique can be found in the text. In the top row, the projection direction is parallel to the net angular momentum vector of all gas within two per cent of the virial radius; in the bottom row, the projection direction is normal to that vector. Each image is $100 \mathrm{pc}$ on a side. Labels indicate the projected half-mass radius of the stars in each galaxy.

that have Pop II stars but do not have any Pop III star (about 13 objects). Hence, either they are polluted with metals by galactic winds from nearby galaxies, or the Pop III star was ejected. These bound objects are likely an example of "triggered" star formation induced by star formation in more massive $\left(10^{8} \mathrm{M}_{\odot}\right)$ halos (Smith et al. 2015). Some of these objects are sufficiently compact to be candidate GCs. Their metallicity is also consistent with their identification as progenitors of today's old GCs.

Five of the 10 most compact objects $\left(r_{h}<7 \mathrm{pc}\right)$ are dark matter dominated and in halos with $M_{d m} \sim 10^{8} \mathrm{M}_{\odot}$. The other five are baryon dominated with $M_{d m}<10^{6} \mathrm{M}_{\odot}$. Excluding halos without Pop II stars (that would be dark today), compact luminous objects are found to have a bimodal mass distribution.

The right panel of Fig. 5 is the same as the left except the color coding shows the ratio of the kinetic to gravitational binding energy of the stars in each object. Galaxies with $r_{\mathrm{h}} \sim 1-2 \mathrm{pc}$ and $r_{\mathrm{h}} \sim 50-200$ pc appear to be bound $\left(\left|E_{K} / E_{W}\right|<1\right)$ and close to virial equilibrium $\left(\left|E_{K} / E_{W}\right| \sim 1 / 2\right)$. Objects with intermediate radii, $r_{\mathrm{h}}$ $\sim 10-40 \mathrm{pc}$, tend to be more loosely bound and further from virial equilibrium $\left(\left|E_{K} / E_{W}\right| \sim 1-2\right)$, suggesting that these stellar systems have not yet reached an equilibrium configuration and that their half-mass radii might still be expanding as a result of gas mass loss in young star-forming clusters.

Fig. 6 shows the mass-to-light ratio $M_{\text {tot }}\left(<r_{\mathrm{h}}\right) / M_{*}(<$ $r_{\mathrm{h}}$ ), (where $M_{\text {tot }}\left(<r_{\mathrm{h}}\right)$ is the dynamical mass within $r_{\mathrm{h}}$ ) as a function of the total dynamical mass. The plot shows two separated set of points: those with $M_{\text {tot }}(<$ 

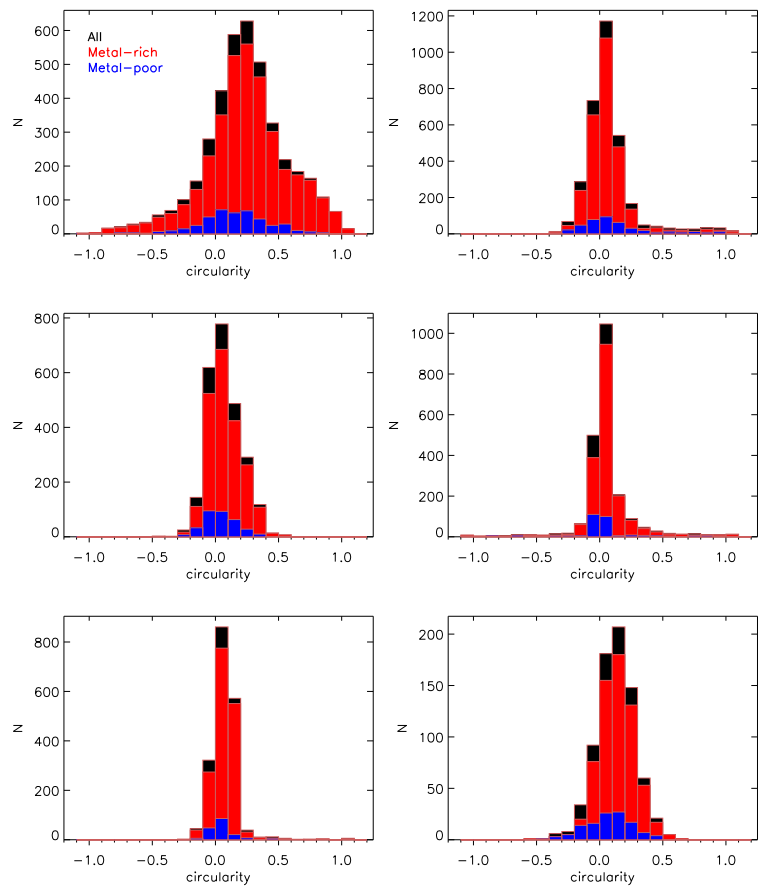

Figure 3. The distribution of orbital circularities (as defined in Equation (2) for stars in the six brightest galaxies in our REF simulation. Blue and red histograms correspond to metal-poor and metal-rich stars respectively, divided at $[\mathrm{Fe} / \mathrm{H}]=-1.5$.

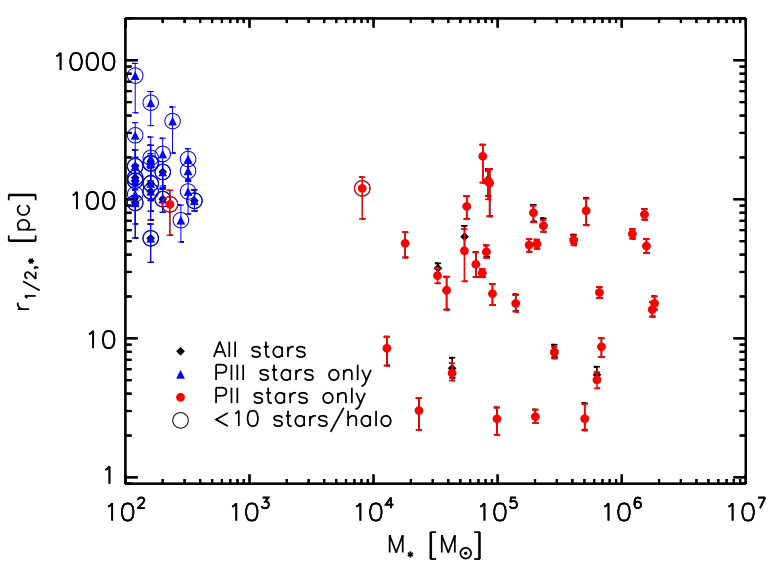

Figure 4. Stellar half-mass radius as a function of stellar mass for all galaxies with more than five star particles in the REF simulation at $z=9$. The projected radii were computed in 100 different random orientations; error bars indicate the $10^{\text {th }}$ and $90^{\text {th }}$ percentiles of the distribution in each case. The halos clustered in the upper left corner of the figure are halos containing only Population III stars. Halos typically obtain multiple Population III stars from accretion of multiple sub-halos containing single Population III stars with $M_{*}=40 \mathrm{M}_{\odot}$.

$\left.r_{\mathrm{h}}\right) / M_{*}\left(<r_{\mathrm{h}}\right) \sim 10^{4}$ are dark matter halos containing only Pop III stars (typically a few stars as indicated by their total stellar masses of $\left.M_{*} \sim 40-200 \mathrm{M}_{\odot}\right)$. The second group of objects have either a constant mass-to-light ratio of a few (the lower horizontal branch in the plot) or a mass-to-light ratio that increases with dynamical mass from ten to a few hundred (the upper branch in the plot).
This indicates that for a fixed dynamical mass of around $10^{7} \mathrm{M}_{\odot}$, we either find dark matter dominated and low surface brightnesses objects (analogous to the ultra-faint dwarfs), or compact star clusters in which the dark matter is a subdominant component of the dynamical mass, but which still can be embedded into larger dark matter halos.

\subsection{Stellar Radii and Sub-grid Star Formation Efficiency}

Fig. 7 is similar to Fig. 4 but for three simulations adopting different values of $\epsilon_{*}$ (sub-grid star formation efficiency) and with color coding of the points showing the ratio of the kinetic to the gravitational binding energy of the objects $\left|E_{K} / E_{W}\right|$. Each panel shows the stellar half-mass radii as a function of stellar mass for all bound objects with more than five star particles in the LSFE $\left(\epsilon_{*}=1 \%\right.$, left panel), REF $\left(\epsilon_{*}=10 \%\right.$, center panel) and $\operatorname{HSFE}\left(\epsilon_{*}=100 \%\right.$, right panel $)$ simulations. The grayscale shaded regions show the stellar to dark matter mass ratio within $r_{h}$, assuming for estimating the dark matter mass within $r_{h}$ a NFW profile for a halo of mass $10^{8} \mathrm{M}_{\odot}$ and virial radius $2 \mathrm{kpc}$, that is rapresentative of typical halos at $z=9$ in $(1 M p c)^{3}$ volume. This is to illustrate that diffuse objects are dark matter dominated while compact objects are baryon dominated. Objects for which the ratio $\left|E_{K} / E_{W}\right|$ is greater than unity (unbound systems) are represented by filled diamond symbols, all other objects by filled circle symbols. We find qualitatively similar bound objects in the three cases, independent of the assumed star formation efficiency (even though it changes by a factor of 100 between the simulations). The spread of the $r_{\mathrm{h}}$ distribution for a given mass (i.e., compact and low surface brightness galaxies) is found in all the simulations, indicating that feedback effects determine the interruption of star formation in the proto-star clusters. We do observe, however, a weak dependence of galaxy luminosity on $\epsilon_{*}$. The luminosity of dwarfs increases by about a factor of two when $\epsilon_{*}$ is increased by a factor of 100 . In the HSFE simulation about 9-11 objects are as compact as GCs, and about 20 are similar to UFDs, with low surface brightness and $r_{\mathrm{h}}$ $\sim 100-200$ pc.

\subsection{Star Formation Histories and Metallicities}

In this subsection we investigate whether the compact dwarfs resembling proto-GCs (based on morphology and kinematics), are also consistent in terms of the metallicity distribution of their stars. GCs are usually seen to have a rather uniform $[\mathrm{Fe} / \mathrm{H}]$ abundance in the range $[\mathrm{Fe} / \mathrm{H}]=-2$ to -1 , indicative of a single stellar population with little or no self-enrichment (e.g., Gratton et al. 2004 Carretta et al. 2009). Thus, to first order approximation, their metallicity reflects the pre-enrichment of their host galaxy. In Fig. 8 we show the distribution of star particle metallicities for six objects in the REF simulation. Note that we advect only one metallicity field, thus $[\mathrm{Fe} / \mathrm{H}]$ is a proxi for the total metal enrichment from SNe. The panels are ordered based on stellar half-mass radii (from the smallest to the largest). The stellar half-mass radius, $r_{\mathrm{h}}$, is included as a label, along with the total stellar mass. The dashed vertical lines indicate the critical metallicity for transition from 

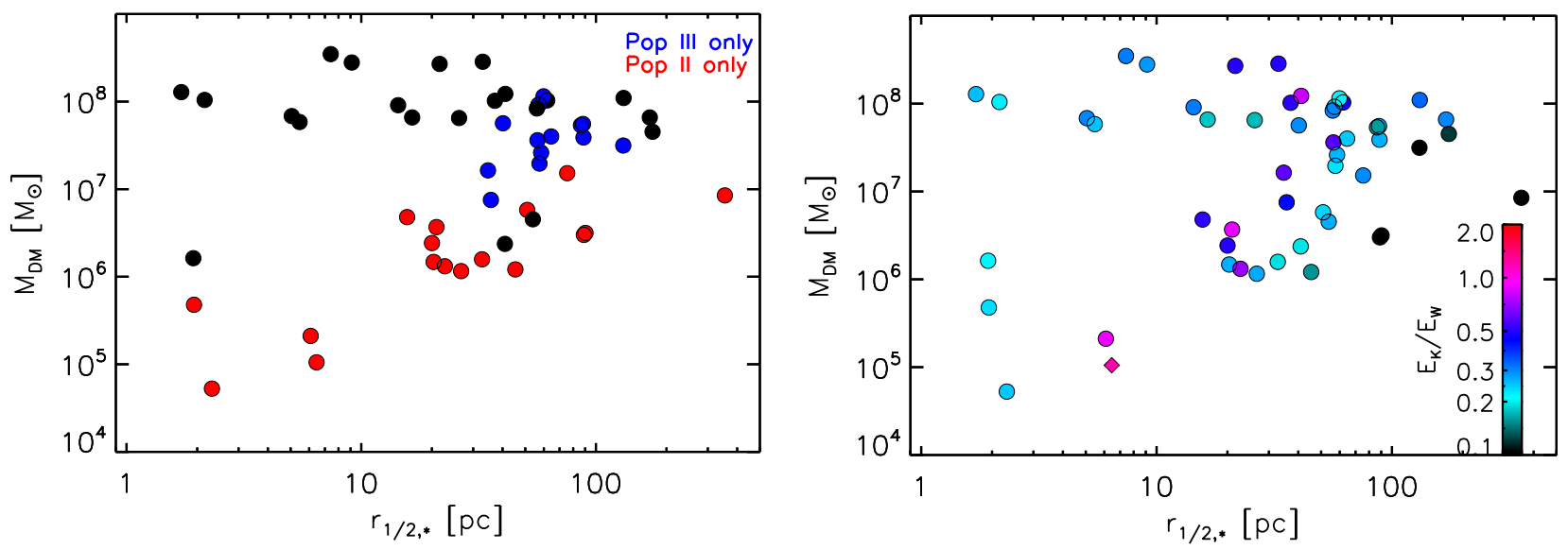

Figure 5. (Left.) Dark matter mass associated with galaxies or compact stellar clusters as a function of their stellar half-mass radius for all bound objects with more than five star particles in the REF simulation. Dark matter halos containing both Population II and Population III stars are shown as black points, halos with only Population III stars are shown in blue, and bound objects with only Population II stars (pre-enriched by external SNe) are shown as red points. The small masses in dark matter $\left(10^{5}-10^{7} \mathrm{M}_{\odot}\right)$ of the bound objects with only Population II stars suggest the existence of a mode of triggered star formation in satellite halos of the first galaxies, leading to the formation of compact star clusters, possibly bound. (Right.) Same as the left panel but with color coding showing the ratio of the kinetic to gravitational binding energy of the system.

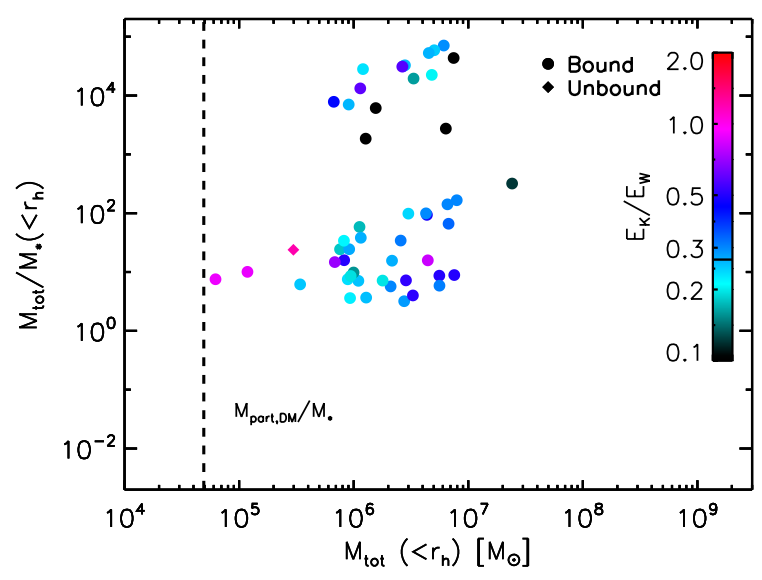

Figure 6. Pseudo-mass-to-light ratio $\left(M_{\text {tot }}\left(<r_{\mathrm{h}}\right) / M_{*}\left(<r_{\mathrm{h}}\right)\right.$ as a function of dynamical mass $M_{\mathrm{tot}}\left(<r_{\mathrm{h}}\right)$ for all galaxies with more than five star particles in the same simulation as in Fig. 5

Pop III to Pop II star formation adopted in our simulations. The most compact object in the figure resembles today's old GCs: it has $r_{\mathrm{h}} \approx 2.7 \mathrm{pc}, M_{*} \approx 5 \times 10^{5} \mathrm{M}_{\odot}$ and a delta-function-like metallicity distribution peaked at $[\mathrm{Fe} / \mathrm{H}]=-1.5$. However, some compact stellar clusters in the simulation (for instance, the one shown in the top-right panel of Fig. 8) have half-mass radii and stellar masses consistent with old GCs, but much broader metallicity distributions, with a range $[\mathrm{Fe} / \mathrm{H}]=-4$ to $[\mathrm{Fe} / \mathrm{H}]=-1$ and therefore it would be classified as an ultra-compact dwarf. These are likely formed as the result of the merger of at least three or four smaller star clusters with different metallicities. Thus, it is rather difficult to distinguish between a GC and the nucleus of an ultra-compact dwarf galaxy solely based on morphology. Indeed, some candidate proto-GCs in our simulations are similar to the nuclei of compact dwarfs because they form at the center of low mass dark matter halos. Most simulated dwarf galaxies with sizes $>10$ pc have rather broad metallicity distributions, very similar to those observed in UFDs and classical dSphs. We also find simulated dwarf galaxies with intermediate sizes $(15-50 \mathrm{pc})$ and low stellar masses, much like the faintest UFDs found around the Milky Way at distances of $\lesssim 150 \mathrm{kpc}$. Tidal stripping of stars has been suggested as the reason for the small sizes of these observed faint dwarfs (Bovill \& Ricotti 2011b), but our simulated dwarfs are already compact when they form, suggesting that need not be the case.

Dwarfs with low stellar masses have metallicity distributions characterized by several minor peaks, with the most prominent typically at the highest metallicity. This suggests that the stellar population is produced by in-situ formation or merger of a handful of small star clusters with relatively narrow metallicity distributions. This scenario is consistent with the rather bursty and spatially segregated mode of star formation observed in most simulated galaxies. A smooth and continuous mode of star formation and self-enrichment is rarely observed in our simulations; a test of our model would be to compare it to observations of the chemical and kinematic signatures typical of star formation taking place in distinct star clusters (Bland-Hawthorn et al. 2015: Webster et al. 2016). Finally, even though the critical metallicity assumed for the transition to Pop II star formation is $[\mathrm{Fe} / \mathrm{H}]=-5$, the lowest metallicities of Pop II stars are between $[\mathrm{Fe} / \mathrm{H}]=-4$ and $[\mathrm{Fe} / \mathrm{H}]=-3$. We have run some simulations with different values of the critical metallicity (see PRG16) and have found very little change in the distribution of stellar metallicities.

Fig. 9 shows the metallicities and formation times of stars in four galaxies in the REF simulation. While the star formation histories of each galaxy are a complex function of many factors, including merger history, halo mass and the local radiation field, these four halos are broadly representative of the galaxy ensemble as a whole. Each color corresponds to an independent progenitor galaxy which hosted the star particle when it formed. Progenitor colors are ordered by the stellar 

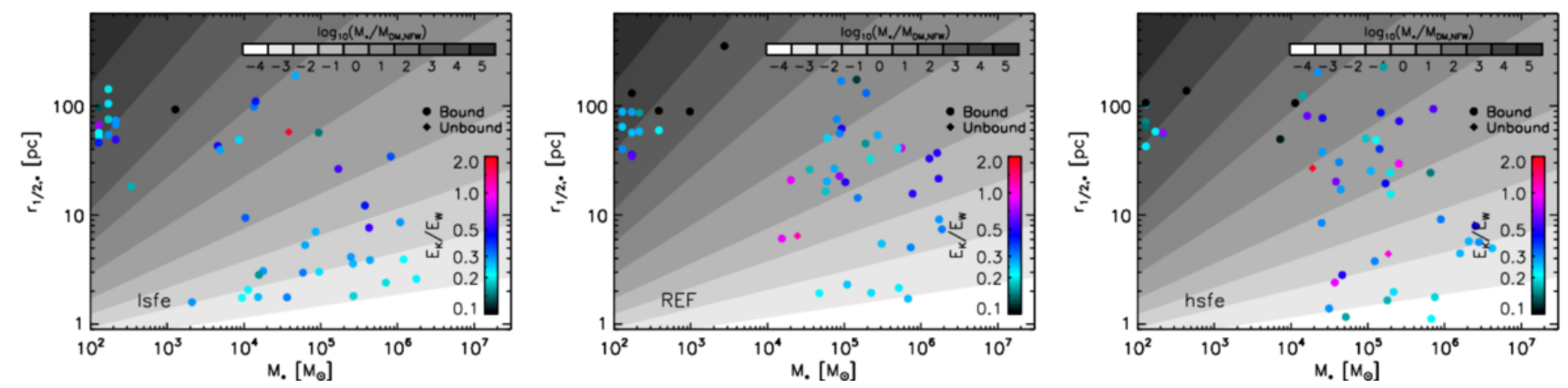

Figure 7. Galaxy sizes for three different values of the Pop II star formation efficiency, $\epsilon_{*}$. See the text for an explanation of the colors and gray-scale regions.

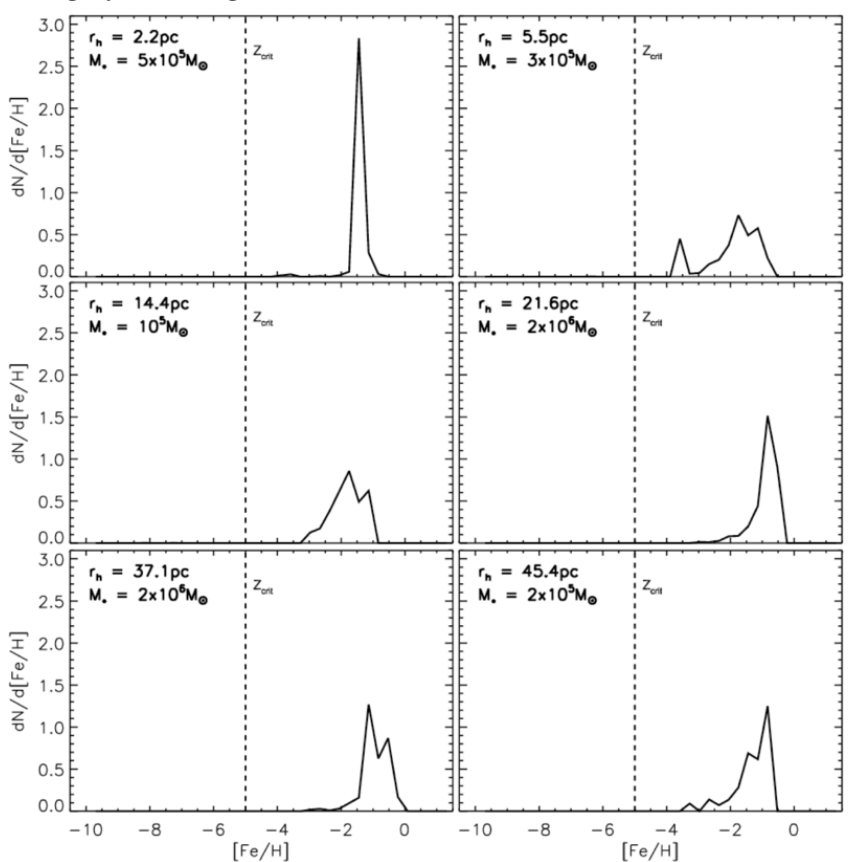

Figure 8. Distribution of star particle metallicities for six galaxies in the REF simulation. The panels are in increasing order of stellar half-mass radius, the value of which is included as a label, along with total stellar mass.

mass contributed, as indicated by the labels in the bottom left of each panel. Star particles were mapped to progenitor galaxies by determining their host halo in the first output after they formed and then associating each halo with a branch of the merger tree. Merger trees were constructed as described in Section 2.5. The stellar halfmass radius of each galaxy is listed in the top left corner of each panel, along with the total stellar mass. The four examples shown suggest an inverse correlation between stellar size and the fraction of stars formed late in the simulation, which is borne out in the galaxy population as a whole. Each galaxy typically contains between two and eight Pop III stars, with many progenitors forming just a single Pop III star. Multiple "tracks" are evident in many of the most massive galaxies, most obviously in the bottom right panel of Fig. 9. The varying gradients of the tracks demonstrate different rates of enrichment in independent progenitor halos. We note that modifying the parameter used to distinguish between Pop III and Pop II stars $\left(z_{\text {crit }}\right)$ affects the size of the "gap" in metallicity between the most metal rich Pop III star and the most metal poor Pop II star, as well as the time delay between the formation of the first Pop III star and Pop II star formation. This delay occurs when gas is enriched above $z_{\text {crit }}$ before it has reached a high enough density to form Pop II stars. For most choices of the simulation's free parameters the delay has very little impact on the typical star formation and final stellar mass of the galaxy, however a too large $z_{\text {crit }}$ or too strong Pop III feedback can suppress Pop II star formation completely in most galaxies (see PRG16).

\section{DISCUSSION: FROM COMPACT STELLAR CLUSTERS TO ULTRA-FAINT GALAXIES}

In this section we investigate the formation of stellar spheroids and and what determines their sizes. We have identified three processes that may play an important role:

i. The extent of the stellar distribution may simply reflect the extent of the star forming gas distribution. However, we found that the gas settles in disks with radii $50-150 \mathrm{pc}$ and a thickness of $10-20 \mathrm{pc}$. Thus, although all stars form in compact clusters within the disk, if the cluster becomes unbound it expands remaining confined by the gravitational potential of the dark matter halo (that to first approximation is spherical), thus a low surface brightness stellar spheroid with radius significantly larger than the disk thickness is formed (see below for a more quantitative discussion).

ii. The stellar spheroid may increase in size due to repeated mergers that dynamically heat the system. This effect may play a role in increasing the size of an already extended stellar spheroid or stellar halo over a rather long time-scale (from $z=9$ to $z=0$ ) rather than self-gravitating compact clusters. Mergers appear to play a role in setting the size of the stellar spheroid in objects containing only Pop III stars (see objects at the top-left corned in Fig. 4). Halos containing multiple Pop III stars have accreted them from mergers of minihalos containing single stars (we have check for this looking at different time snapshots tracing the merging of satellites). Because Pop III stars are accreted, their velocity dispersion is roughly the virial velocity of the host dark matter halo, or slightly lower due to the effect of dynamical friction. Thus their radial distribution extends to nearly $r_{\max }$.

iii. In our simulations stars form in dense clusters with $3 \mathrm{D}$ velocity dispersion at formation in the range $20-40 \mathrm{~km} \mathrm{~s}^{-1}$. If the gas clump in which the cluster forms converts into stars $<50 \%$ of the gas, 

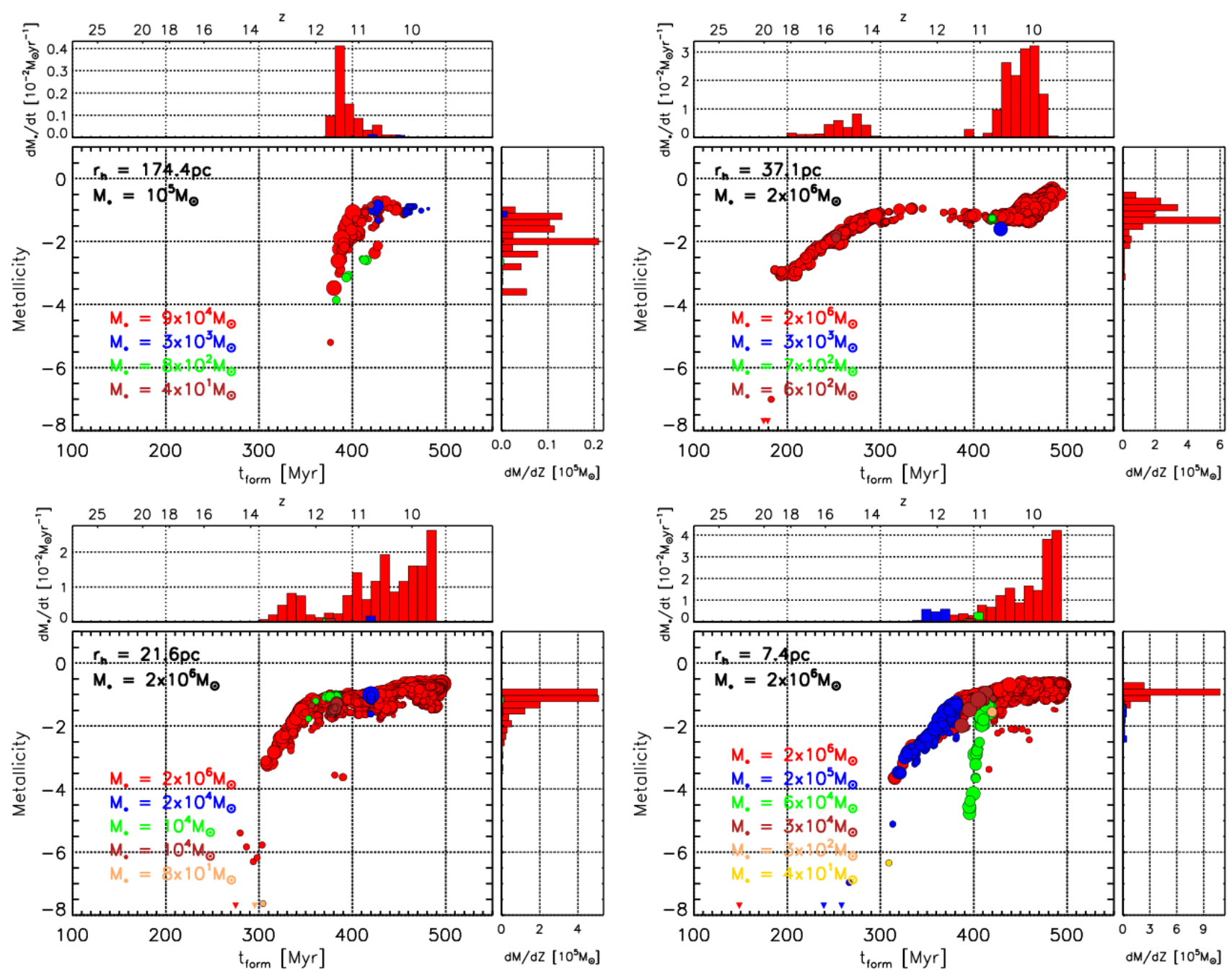

Figure 9. The metallicities and formation times of all star particles belonging to four representative halos in the REF simulation. Triangular points correspond to stars with metallicities below the lower limit of the ordinate axis. Colors indicate the progenitor galaxy in which the star formed, red being the most massive ("main-branch") progenitor. The labels in each panel indicate the stellar half-mass radius and stellar mass (top left legend) and the total mass formed in each progenitor (bottom left legend). Histograms show the star formation rate and metallicity distribution.

the cluster becomes unbound after the unused gas is expelled by SN feedback (see, Hills 1980). Expanding stars with a final velocity dispersion less than $v_{\text {cir }} \sim 20 \mathrm{kms}^{-1}$ remain bound inside the dark matter halos and can extend close to $r_{\max }$.

The large range of radii observed in our simulations and their weak dependence on the SFE, $\epsilon_{*}$, suggest that iii) plays a dominant role.

In the left panel of Fig. 10 we show how stars forming in bursts tend to expand into more diffuse distributions over time. "Clusters" are identified by dividing each galaxy's stars into 5 Myr bins in formation time and then selecting any bins which account for at least 10 per cent of the total stellar mass. While there is no explicit requirement that the stars in each burst be close to each other, the majority have initial half-mass radii $<5$ pc. Each selection is traced forward to subsequent output snapshots and its half-mass radius is recorded. In order to be plotted in Fig. 10, bursts must occur before the penultimate output. We note that a number of the tracks show expansion from $\sim 5$ pc to $\sim 50$ pc over a period of 100 Myr or so, while there is tentative evidence that a few (between 1 to 3 ) remain fairly compact $(<30$ $\mathrm{pc}$ ). In many of the late-forming clusters, it is unclear whether their final sizes will remain close to GCs radii or expand to become similar to UFDs.

We can use a toy model to interpret the data from the simulation. The initial velocity dispersion of the stars in a proto-cluster, $\sigma_{*}^{i c}$, can be estimated from the gas mass in the star forming region of size $r_{h}^{i c} \sim 2$ pc:

$$
\sigma_{*}^{i c} \sim\left(\frac{r_{h}^{i c}}{t_{d y n}}\right) \sim 50 \mathrm{~km} \mathrm{~s}^{-1}\left(\frac{r_{h}^{i c}}{2 p c}\right),
$$

where $t_{\text {dyn }}=\left(G \rho_{\text {gas }}\right)^{-1 / 2} \sim 0.1$ Myr for $\rho_{\text {gas }}=10^{-18}$ $\mathrm{gcm}^{-3}$, which is appropriate at the maximum level of refinement in our simulation (see Fig. 1). Thus, the initial velocity dispersion of the proto-star clusters is $\sigma_{*}^{i c}>v_{\text {cir }} \sim 10-20 \mathrm{~km} \mathrm{~s}^{-1}$, where $v_{\text {cir }}$ is the circular velocity of the dark matter halo. However, if the star cluster is self-gravitating and bound, the stars will not be able to escape the potential of the dark matter halo. 

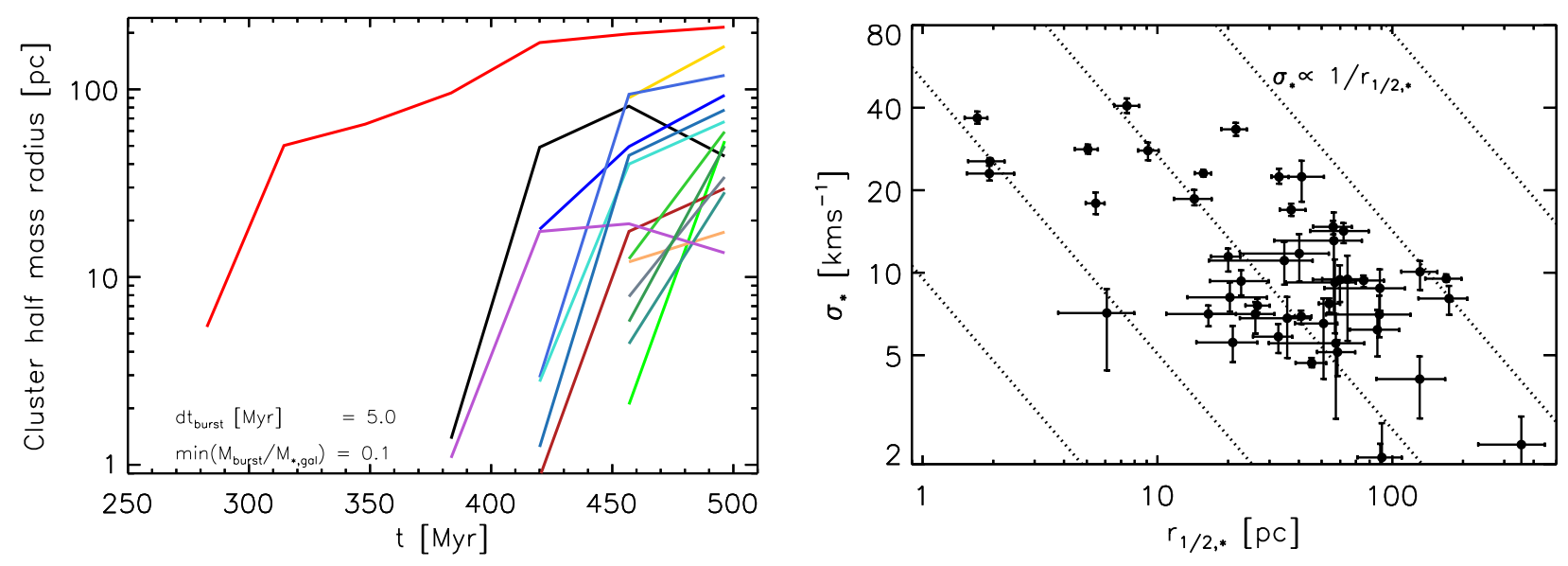

Figure 10. (Left) Size evolution of star bursts (clusters) identified in the REF simulation before the penultimate output (therefore tracks of compact clusters formed recently in the simulation do not appear here). (Right) 3D velocity dispersion of the stars as a function of the stellar half-mass radius for all bound objects with more than five star particles in the REF simulation at $z=9$.

Instead, if the star cluster becomes unbound as a result of gas mass loss, its radius will increase and the velocity dispersion of the stars will decrease.

Next we consider the effect of mass loss on the dynamical evolution of a stellar system (Hills 1980). If the initial mass of the star forming cloud is $M_{\text {gas }}^{c c}$ and the final mass after star formation and gas loss is $M_{*}$, we can define the star formation efficiency in the proto star cluster: $\epsilon_{c l}=M_{*} / M_{\text {gas }}^{i c}$. There are two limiting cases. If $t_{\text {loss }} \ll t_{\text {dyn }}$ (impulsive gas loss):

$$
\begin{aligned}
\frac{r_{h}}{r_{h}^{i c}} & =\frac{\epsilon_{c l}}{2 \epsilon_{c l}-1} \text { with } 0.5<\epsilon_{c l}<1, \\
\frac{\sigma_{*}}{\sigma_{*}^{i c}} & \approx\left(\epsilon_{c l} \frac{r_{h}^{i c}}{r_{h}}\right)^{1 / 2} .
\end{aligned}
$$

In this case, only if $\epsilon_{c l}>50 \%$ will the cluster remain bound. The velocity dispersion of the stars decreases as $\sigma_{*} \propto r_{h}^{-1 / 2}$ as the cluster expands to the new virial equilibrium after mass loss ${ }^{6}$ (for $\epsilon_{c l}=50 \%, r_{h} \rightarrow \infty$ and $\left.\sigma_{*} \rightarrow 0\right)$.

If $t_{\text {loss }} \gg t_{\text {dyn }}$ (quasi-adiabatic expansion):

$$
\begin{aligned}
\frac{r_{h}}{r_{h}^{i c}} & =\frac{1}{\epsilon_{g c}} \text { with } 0<\epsilon_{c l}<1, \\
\frac{\sigma_{*}}{\sigma_{*}^{i c}} & \approx \frac{r_{h}^{i c}}{r_{h}} .
\end{aligned}
$$

In the right panel of Fig. 10 we plot the velocity dispersion of stars, $\sigma_{*}$, as a function of the half-mass radius, $r_{\mathrm{h}}$, for the galaxies in the REF simulation. We observe a bimodal distribution of $\sigma_{*}$ : several galaxies (about 12) are found to have $\sigma_{*} \sim 20-40 \mathrm{~km} \mathrm{~s}^{-1}$ and $r_{\mathrm{h}} \sim 1-20 \mathrm{pc}$, while the rest are concentrated in the parameter space $\sigma_{*} \sim 10 \pm 5 \mathrm{~km} / \mathrm{s}$ and $r_{\mathrm{h}} \sim 100 \pm 80 \mathrm{pc}$. In the plot we also

6 To derive the velocity dispersion we have applied the virial theorem to the final bound configuration, but the equation is nearly identical to Eq. 26 in Hills (1980) for the expansion velocity of unbound associations. show lines with $\sigma_{*} \propto r_{\mathrm{h}}^{-1}$, consistent with quasi-adiabatic expansion of the cluster, as given by Equation (7).

Thus, combining the results illustrated in both panels of Fig. 10, a picture emerges in which the low-surface brightness dwarfs with $r_{\mathrm{h}} \sim 100 \mathrm{pc}$ and $\sigma_{*} \sim 10 \mathrm{~km} \mathrm{~s}^{-1}$ are the (young) descendants of dwarfs galaxies that form their stars in compact clusters with high stellar velocity dispersions. A fraction of these clusters with the highest star formation efficiencies remain bound and resemble today's GCs, ultra-compact dwarfs or dwarf-globular transition objects, while the others expand in the dark matter halo potential until the stellar velocity dispersion (that decreases as $\sigma_{*} \propto r_{h}^{-\alpha}$, with $\alpha \sim 0.5-1$ ) becomes comparable to the halo circular velocity $v_{\text {cir }}$ at the radius $r_{h}$ :

$$
\sigma_{*}\left(r_{h}\right)=v_{\text {cir }}\left(r_{h}\right)
$$

At this point, the cluster is dark matter dominated and bound by the gravitational potential of the dark matter halo. Thus, in this model a range of stellar half-mass radii are possible, depending on the initial $\sigma_{*}^{i c}$ and efficiency of star formation in the cluster $\epsilon_{c l}$. However, $r_{h}$ cannot exceed $r_{\max }$ of the halo (where the circular velocity reaches its maximum value). If this happens, most of the stars will be lost from the dwarf into the IGM.

Assuming the cluster becomes unbound and evolves quasi-adiabatically, and integrating $d r_{h} / d t=\sigma_{*}\left(r_{h}\right)$, obtained from dimensional analysis, we get

$$
\frac{r_{h}(t)}{r_{h}^{i c}}=\left(\frac{t}{t_{\mathrm{dyn}}}\right)^{1 / 2}
$$

where $t_{\text {dyn }} \equiv r_{h}^{i c} / \sigma_{*}^{i c} \sim 0.1$ Myr. Comparing $r_{h}(t)$ in Equation (9) as a function of time with the evolutionary tracks in the left panel of Fig. 10 we find good agreement between our toy model and the simulated clusters.

\subsection{Comparison to Present-day Compact Clusters and Nearby Dwarf Galaxies}

In Figure 11 we show a comparison between the properties of luminous objects in our REF simulation (blue circles) in comparison to Milky Way globular clusters (shown as stars) from Harris (1996) and nearby dwarf 

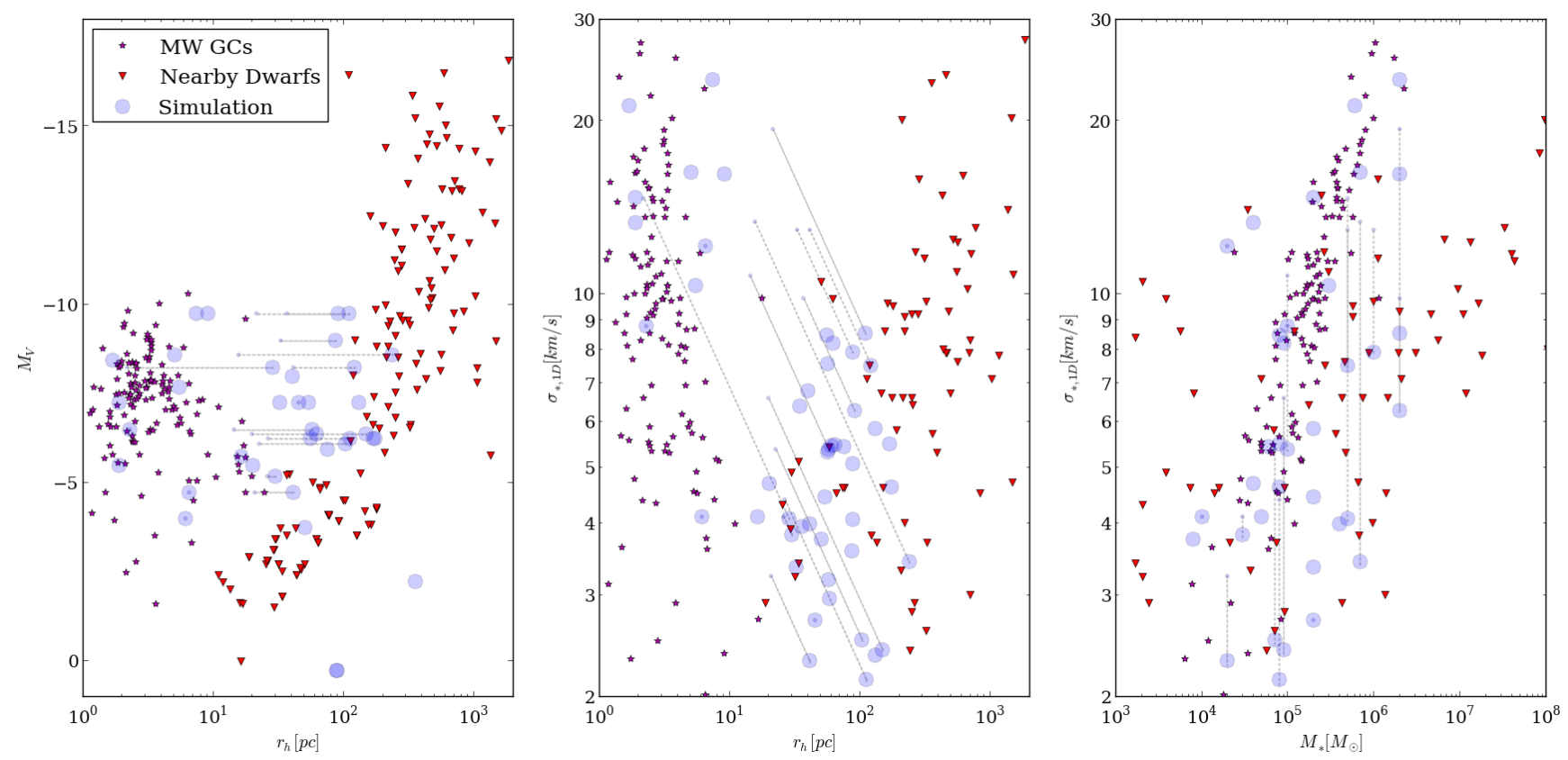

Figure 11. Comparison between simulated compact clusters and dwarf galaxies after a simple extrapolation of stellar properties from $z=9$ to $z=0$ (blue circles), to Milky Way globular clusters (stars) and nearby classical and ultra-faint dwarf galaxies (triangles). We assumed a mass to light $M / L=3$. The dotted segments show the expected evolution of clusters that are either unbound or still have $>50 \%$ of the their mass is in gas (see text). (Left.) Visual absolute magnitude as a function of half-light radius, $r_{h}$. (Center.) Line of sight velocity dispersion as a function of $r_{h}$. (Right.) Line of sight velocity dispersion as a function of stellar mass.

galaxies (shown as triangles), including classical dwarfs (dSphs, dEs, dIrrs) and ultra-faint dwarfs from the McConnachie (2012) compilation. Similar plots for a compilation of GCs and compact dwarfs are shown in KisslerPatig et al. (2006). Since our simulation stops and is analyzed at redshift $z=9$, in order to compare the simulated galaxies to present-day clusters and dwarf galaxies, we assume a mass to light ratio $M / L_{V}=3$, that takes into account mass loss and passive stellar evolution over about 12 Gyrs as in Ricotti \& Gnedin (2005). Several compact clusters in our simulation are recently formed, thus they may not remain bound if i) they are not in virial equilibrium, or ii) they contain a significant amount of gas (> 50\%) within their half-light radius, that if expelled by $\mathrm{SNe}$ of photoevaporation may unbind the cluster (only a couple of objects are in this category). For those objects we evolve their half light radii $r_{h}(t)$ as in Eq. (9) and their velocity dispersions $\sigma_{*}(t)$ as in Eq. (7) until the stars becomes bound by the dark matter halo as in Eq. (8). The dotted lines in the plot show the evolution of such objects, evolving from the small to the larger blue circles at the extremes of the dotted lines. Note that here we plot the line of sight velocity dispersion, $\sigma_{*, 1 D}$, that we simply relate to the $3 \mathrm{D}$ velocity dispersion as $\sigma_{*}=\sqrt{3} \sigma_{*, 1 D}$. Also, in order to calculate the velocity profile $v_{\text {cir }}(r)$ of each halo we assume NFW density profile with concentration parameter $c=4$, that is appropriate for recently virialized halos. We see that many simulated objects that are initially in a region of parameter space devoid of observed objects (a narrow strip laying between GCs and UFDs), are evolving to lower $\sigma_{*, 1 D}$ and larger $r_{h}$ toward the region occupied by UFDs.

The extrapolation of observed properties to $z=0$ is rather simplistic, and is not the main focus of this paper. Using N-body simulations, previous works by Bovill \& Ricotti (2011a b) have looked in detail at several effects that we have neglected. They show that only small mass halos that are rather isolated at $z=9$ evolve to $z=0$ without merging into larger halos and thus accreted fresh gas. A subset of these halos evolving in isolation with maximum circular velocity $v_{\max }<20-25 \mathrm{~km} / \mathrm{s}$ will not accrete fresh gas from the IGM after reionization at $z \lesssim 6-9$. These object, are suitable for a direct comparison to UFDs and are what has been defined "fossil" galaxies (Ricotti \& Gnedin 2005, Bovill \& Ricotti 2009). Bound compact cluster may also survive intact for about a Hubble time (13.6 Gyrs) only if they are more massive than $10^{4} \mathrm{M}_{\odot}$ (e.g., Katz \& Ricotti 2014). If their mass is instead smaller, the time scale for evaporation due to 2-body encounters is shorter than the Hubble. This is interesting because the faintest UFDs have $M_{*} \sim 10^{3}-10^{4} \mathrm{M}_{\odot}$, thus they could be formed by the secular evaporation of compact clusters residing in a small mass dark matter halo. As discussed above, the cluster will puff up until the stars become bound by the potential of the dark matter halo, as in Eq. (8). Of course GCs can be destroyed by tidal stripping and shocks while interacting with their host galaxy . Roughly, only 1 or 2 in 10 GCs is expected to survive within the Milky Way over a Hubble time (Katz \& Ricotti 2014).

Fig 12 shows the mass in gas within the half-light radius, $M_{\text {gas }}$, normalized by the total baryonic mass $M_{*}+M_{\text {gas }}$ as a function of $r_{h}$. In compact clusters with $r_{h}<10 \mathrm{pc}$ the mass in gas is less than the mass in stars, while in larger objects the mass of the stars is sub dominant with respect to the gas mass (and the dark matter mass). The points shown as diamonds are unbound 


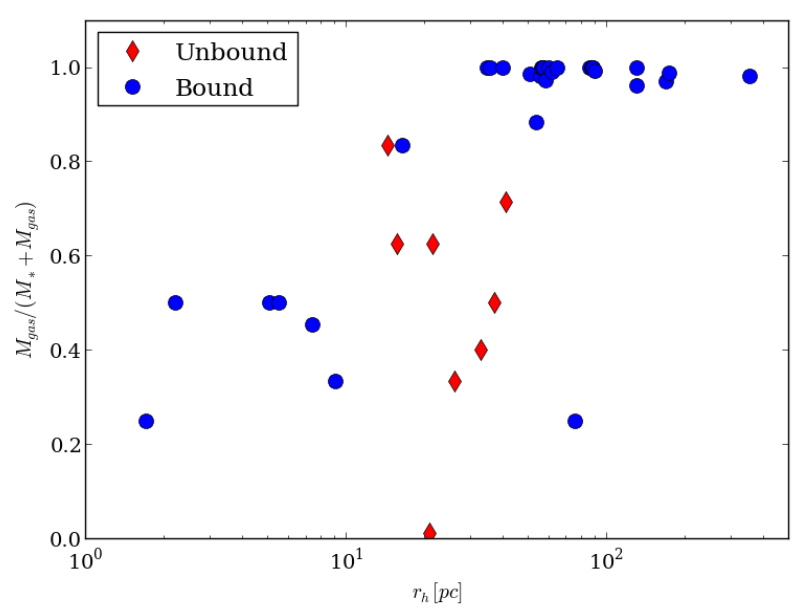

Figure 12. Gas mass within the half-light radius, $M_{\text {gas }}$, normalized by the total baryonic mass $M_{*}+M_{\text {gas }}$ as a function of $r_{h}$. The circles show bound object and the diamonds unbound objects.

objects, thus are transitioning toward larger $r_{h}$, while the circles show bound objects (bound by the baryons if $r_{h}<10 \mathrm{pc}$ and by the dark matter for $r_{h}>30 \mathrm{pc}$ ). The figure shows that in compact clusters either $>50 \%$ of the gas has been used for star formation (i.e., the star formation efficiency in the proto-cluster is $\epsilon_{c l}>50 \%$ ), or $\epsilon_{c l}<50 \%$ and part of the gas has been expelled by radiation and $\mathrm{SN}$ feedback. In the former case $\left(\epsilon_{c l}>50 \%\right)$ the cluster remains bound while in the second case it will become unbound once all the gas is lost, and it will expand untill $\sigma(r)=v_{c i r}(r)$ (see $\S$ 4). The figure shows that radiation and $\mathrm{SN}$ feedback did not clear out all the gas withing the dark matter halo of most objects with $r_{h}>30 \mathrm{pc}$, as they are gas rich. However, the star formation rate in galaxies is self-regulated by feedback (see $\S 3.5$ and Fig. 7) and the range of $\epsilon_{c l}$ found in the simulation is determined by the effectivness of feedback in terminating star formation in star forming gas clumps and by their density (the sub-grid star formation law we use converts into stars $\epsilon_{*}=10 \%$ of the gas in a cell per local free-fall time). We should however note that with a spatial resolution of $1 \mathrm{pc}$, the internal structure of young compact star clusters $\left(r_{h} \sim\right.$ few $\left.-10 \mathrm{pc}\right)$ is only marginally resolved. Higher resolution simulations are needed to confirm quantitative results in the simulation. For instance the number of bound stellar clusters may increase by increasing the numerical resolution.

Multiple stellar populations are present in all globular clusters observed to date (e.g., Gratton et al.|2004; D'Ercole et al. 2008, Carretta et al. 2010). Fig. 9 shows that when we look in detail at the stellar populations of compact star clusters, several have stars with distinct metallicities and formation times. Some compact clusters form in very low mass statellites orbiting the host dwarf galaxy (offset from center of their host), triggered by external metal enrichment from galactic winds. Others are found close to the center of their host dark matter halos, resambling in terms of their metallicity the nuclei of compact dEs. These second type of compact clusters should be able to form stars in multiple bursts still remaining compact, due to gas fallback in the gravitational potential of the dark matter halo. We do not have the necessary resolution to study in detail the process of SN feedback and gas fall back, but is an idea worth exploring with dedicated high-resolution simulations.

\section{SUMMARY AND CONCLUSIONS}

We have presented results from cosmological simulations of the formation of the first stars and galaxies performed with an adaptive mesh hydrodynamics code. The code includes recipes for Pop III and Pop II star formation, self-consistent radiative transfer and a model for the formation and dissociation of molecular hydrogen, allowing us to resolve internal structure within the molecular clouds of individual galaxies.

We find that the first galaxies have thick gaseous disks but the stars form spheroids with half light radii larger than the disk thickness. Within the disk, star formation takes place in compact star clusters with initial velocity dispersions $\sigma_{*} \sim 20-40 \mathrm{~km} / \mathrm{s}$ and half-mass radii of a few parsecs. However, due to gas loss, most of the clusters become unbound and expand, until the stars become bound again by the gravitational potential of the dark matter halo. This is confirmed by our analysis of the simulations showing that the stars in low surface brightness dwarf galaxies were in much denser star clusters at the time of their formation. These clusters had sufficiently high initial velocity dispersions to expand to $\sim 100 \mathrm{pc}$ in a few Myrs after gas loss following SN and radiation feedback. Thus, the main reason for the formation of stellar spheroids with little rotation in the first dwarfs appears to be the high velocity dispersion of stars in the proto star clusters, relative to the circular velocity of the host halo $\left(10-20 \mathrm{~km} \mathrm{~s}^{-1}\right)$. There is some tentative evidence that we have captured the formation of the first bound compact stellar systems (although is unclear their classification as proto-GCs, ultra-compact dwarfs or dwarf-globular transition objects), as about 1 to 3 of the clusters with initially compact configuration remain bound for a few hundred Myrs, until the end of the simulation at $z \sim 9$. Higher resolution simulations evolved to lower redshift are necessary to confirm this result. However, the origin of low surface brightness dwarfs as evaporated compact stellar clusters appears robust, suggesting a new connection between UFDs (or dSphs) and compact stellar system. We therefore speculate that:

i. A fraction of old GCs around the Milky Way, although clearly not all of those observed, may form within dark matter satellites around low mass halos in the early universe (see triggered star formation in $\S 3.4$ and Figure 5 .

ii. Observational signatures of a dark matter minihalo surrounding GCs (in addition to dynamical evidence for dark matter in the outer parts of the cluster) would include: an enhanced ability to retain metals during their formation and possible modifications to the effects of dynamical friction and tides on their secular evolution.

iii. Candidate compact clusters are offset from the center of their host halos by an average of $\sim 40 \mathrm{pc}$. Some are found in $10^{8} \mathrm{M}_{\odot}$ halos that contain both Pop III and Pop II stars, but a few are found in very low mass $\left(10^{6} \mathrm{M}_{\odot}\right)$ halos that do not contain Pop III stars. This indicates that the formation 
of bound stellar clusters was triggered by the influence of a nearby luminous galaxy. Their formation in $10^{6} \mathrm{M}_{\odot}$ satellites of $10^{8} \mathrm{M}_{\odot}$ halos may help explain the spatial distribution of GCs in nearby dwarf galaxies.

iv. In our simulations UFDs originate from the dissolution of only a few distinct star clusters. Open clusters, or compact clusters with masses $\lesssim 10^{4} \mathrm{M}_{\odot}$ (that evaporate in a Hubble time due to 2-body encounters) will expand inside the dark matter halo, resulting in a low surface brightness, dark matter dominated galaxy. It may be possible to identify stars that originated in the distinct progenitor clusters through their chemical and kinematic properties.

v. The faintest dwarfs found within $150 \mathrm{kpc}$ of the Milky Way have half-mass radii of $20-40$ pc and hence are typically less extended, with higher surface brightnesses than some brighter satellites. In our simulations such dwarf galaxies, with properties intermediate between $r_{\mathrm{h}} \sim 100$ pc UFDs and GCs exist. The stellar half light radii of these objects are set by the initial velocity dispersion of the stars in the proto-cluster and the gas mass loss rate. However, it is less clear if the fossil remnants of these compact objects will remain stable to the present (for 12 Gyr), or if their stellar component will expand further during their secular evolution and interaction with Milky Way.

vi. The metallicity and duration of star formation in some compact stellar clusters are consistent with a single star burst. However, several compact dwarfs with sizes $\sim 5-40$ pc have one or more additional subdominant stellar components. These superimposed components are typical of dwarf galaxy formation in the early universe as illustrated in Fig. 9 a rather bursty and spatially segregated star formation history spread over a few $100 \mathrm{Myr}$, and a metallicity distribution produced by the hierarchical assembly of several smaller dwarfs (Ricotti \& Gnedin 2005).

The simulations presented here and in PRG16 are only a first step to understand the emergence of the first light in the universe. The qualitative results are intriguing because they suggest interesting ideas on the origin of compact star clusters and ultra-faint dwarfs and a deeper physical understanding of their connections. However, in order to answer questions on the statistics of the relics of the first objects in the Local Group, a larger computational volume and higher dark matter resolution will be necessary to achieve numerical convergence.

\section{ACKNOWLEDGMENTS}

OHP and MR acknowledge support from NASA grant NNX10AH10G and NSF grant CMMI1125285. The authors acknowledge the University of Maryland supercomputing resources (http://www.it.umd.edu/hpcc) made available in conducting the research reported in this paper. This work also used the Extreme Science and Engineering Discovery Environment (XSEDE), which is supported by NSF grant number ACI-1053575.

\section{REFERENCES}

Abadi, M. G., Navarro, J. F., Steinmetz, M., \& Eke, V. R. 2003, ApJ, 591, 499

Abel, T., Bryan, G. L., \& Norman, M. L. 2000, ApJ, 540, 39

—. 2002, Science, 295, 93

Barkana, R., \& Loeb, A. 1999, ApJ, 523, 54

Belokurov, V., Zucker, D. B., Evans, N. W., et al. 2007, ApJ, 654, 897

Belokurov, V., Walker, M. G., Evans, N. W., et al. 2010, ApJ, 712, L103

Benitez-Llambay, A., Navarro, J. F., Abadi, M. G., et al. 2014 ArXiv:1405.5540, arXiv:1405.5540

Bland-Hawthorn, J., Sutherland, R., \& Webster, D. 2015, ApJ, 807,154

Bovill, M. S., \& Ricotti, M. 2009, ApJ, 693, 1859

—. 2011a, ApJ, 741, 17

-. 2011b, ApJ, 741, 18

Boylan-Kolchin, M., Bullock, J. S., \& Garrison-Kimmel, S. 2014, ArXiv e-prints, arXiv:1405.1040

Boylan-Kolchin, M., Weisz, D. R., Johnson, B. D., et al. 2015, MNRAS, 453, 1503

Bromm, V., Coppi, P. S., \& Larson, R. B. 1999, ApJ, 527, L5

Bromm, V., Ferrara, A., Coppi, P. S., \& Larson, R. B. 2001, MNRAS, 328, 969

Brown, T. M., Tumlinson, J., Geha, M., et al. 2012, ApJ, 753, L21 -. 2014, ApJ, 796, 91

Carretta, E., Bragaglia, A., Gratton, R., D’Orazi, V., \&

Lucatello, S. 2009, A\&A, 508, 695

Carretta, E., Bragaglia, A., Gratton, R. G., et al. 2010, A\&A, 516, A55+

Chabrier, G. 2003, PASP, 115, 763

Christensen, C., Quinn, T., Governato, F., et al. 2012, MNRAS, 425,3058

Ciardi, B., Ferrara, A., Governato, F., \& Jenkins, A. 2000,

MNRAS, 314, 611

Clark, P. C., Glover, S. C. O., \& Klessen, R. S. 2008, ApJ, 672, 757

Davis, M., Efstathiou, G., Frenk, C. S., \& White, S. D. M. 1985 ApJ, 292, 371

D'Ercole, A., Vesperini, E., D'Antona, F., McMillan, S. L. W., \& Recchi, S. 2008, MNRAS, 391, 825

Drlica-Wagner, A., Bechtol, K., Rykoff, E. S., et al. 2015, ApJ, 813, 109

Efstathiou, G. 1992, MNRAS, 256, 43P

Forbes, D. A., Pota, V., Usher, C., et al. 2013, MNRAS, 435, L6

Geyer, M. P., \& Burkert, A. 2001, MNRAS, 323, 988

Gnedin, N. Y. 2000, ApJ, 535, 530

-. 2008, ApJ, 673, L1

—. 2014, ArXiv e-prints, arXiv: 1403.4245

Gnedin, N. Y., \& Abel, T. 2001, New Astronomy, 6, 437

Gnedin, N. Y., \& Kravtsov, A. V. 2011, ApJ, 728, 88

Gnedin, N. Y., Tassis, K., \& Kravtsov, A. V. 2009, ApJ, 697, 55

Gnedin, O. Y., Brown, W. R., Geller, M. J., \& Kenyon, S. J. 2010, ApJ, 720, L108

Gratton, R., Sneden, C., \& Carretta, E. 2004, ARA\&A, 42, 385

Greif, T. H., Springel, V., White, S. D. M., et al. 2011, ApJ, 737, 75

Harris, W. E. 1996, AJ, 112, 1487

Hills, J. G. 1980, ApJ, 235, 986

Hoeft, M., Yepes, G., Gottlöber, S., \& Springel, V. 2006, MNRAS, 371, 401

Hopkins, P. F., Kereš, D., Oñorbe, J., et al. 2014, MNRAS, 445, 581

Hopkins, P. F., Quataert, E., \& Murray, N. 2012, MNRAS, 421, 3488

Jaacks, J., Thompson, R., \& Nagamine, K. 2013, ApJ, 766, 94

Johnson, J. L., Dalla Vecchia, C., \& Khochfar, S. 2013, MNRAS, 428,1857

Karlsson, T., Bland-Hawthorn, J., Freeman, K. C., \& Silk, J. 2012, ApJ, 759, 111

Katz, H., \& Ricotti, M. 2013, MNRAS, 432, 3250

-. 2014, MNRAS, 444, 2377

Katz, H., Sijacki, D., \& Haehnelt, M. G. 2015, MNRAS, 451, 2352

Kimm, T., \& Cen, R. 2014, ApJ, 788, 121

Kissler-Patig, M., Jordán, A., \& Bastian, N. 2006, A\&A, 448, 1031 
Koposov, S. E., Belokurov, V., Torrealba, G., \& Evans, N. W. 2015, ApJ, 805, 130

Kravtsov, A. V. 1999, PhD thesis, New Mexico State University —. 2003, ApJ, 590, L1

Kravtsov, A. V., Berlind, A. A., Wechsler, R. H., et al. 2004, ApJ, 609, 35

Kravtsov, A. V., Klypin, A. A., \& Khokhlov, A. M. 1997, ApJS, 111,73

Kuhlen, M., Krumholz, M. R., Madau, P., Smith, B. D., \& Wise, J. 2012, ApJ, 749, 36

Kuhlen, M., Madau, P., \& Krumholz, M. R. 2013, ApJ, 776, 34

Lada, C. J., \& Lada, E. A. 2003, ARA\&A, 41, 57

Leitherer, C., Schaerer, D., Goldader, J. D., et al. 1999, ApJS, 123,3

Maio, U., Ciardi, B., Dolag, K., Tornatore, L., \& Khochfar, S. 2010, MNRAS, 905

Martin, N. F., de Jong, J. T. A., \& Rix, H. 2008, ApJ, 684, 1075

McConnachie, A. W. 2012, AJ, 144, 4

Muratov, A. L., Gnedin, O. Y., Gnedin, N. Y., \& Zemp, M. 2013a, ApJ, 772, 106

-. 2013b, ApJ, 773, 19

Okamoto, T., Gao, L., \& Theuns, T. 2008, MNRAS, 390, 920

O'Shea, B. W., Wise, J. H., Xu, H., \& Norman, M. L. 2015, ApJ, 807, L12

Pawlik, A. H., Milosavljević, M., \& Bromm, V. 2011, ApJ, 731, 54

Pawlik, A. H., Schaye, J., \& van Scherpenzeel, E. 2009, MNRAS, 394, 1812

Polisensky, E., \& Ricotti, M. 2011, Phys. Rev. D, 83, 043506

Press, W. H., \& Davis, M. 1982, ApJ, 259, 449

Ricotti, M. 2002, MNRAS, 336, L33

-. 2009, MNRAS, 392, L45

Ricotti, M., \& Gnedin, N. Y. 2005, ApJ, 629, 259

Ricotti, M., Gnedin, N. Y., \& Shull, J. M. 2002a, ApJ, 575, 33

-. 2002b, ApJ, 575, 49

-. 2008a, ApJ, 685, 21

Ricotti, M., \& Ostriker, J. P. 2004, MNRAS, 350, 539

Ricotti, M., Ostriker, J. P., \& Mack, K. J. 2008b, ApJ, 680, 829

Rudd, D. H., Zentner, A. R., \& Kravtsov, A. V. 2008, ApJ, 672, 19

Sawala, T., Frenk, C. S., Fattahi, A., et al. 2014, ArXiv:1406.6362, arXiv:1406.6362
Scannapieco, C., Wadepuhl, M., Parry, O. H., et al. 2012, MNRAS, 423, 1726

Scannapieco, E., Schneider, R., \& Ferrara, A. 2003, ApJ, 589, 35

Schaerer, D. 2002, A\&A, 382, 28

Schneider, R., Salvaterra, R., Ferrara, A., \& Ciardi, B. 2006, MNRAS, 369, 825

Simpson, C. M., Bryan, G. L., Johnston, K. V., et al. 2013, MNRAS, 432, 1989

Smith, B. D., Wise, J. H., O'Shea, B. W., Norman, M. L., \& Khochfar, S. 2015, MNRAS, 452, 2822

Springel, V., White, S. D. M., Tormen, G., \& Kauffmann, G. 2001, MNRAS, 328, 726

Tassis, K., Gnedin, N. Y., \& Kravtsov, A. V. 2012, ApJ, 745, 68

Thompson, R., Nagamine, K., Jaacks, J., \& Choi, J.-H. 2014, ApJ, 780, 145

Tomassetti, M., Porciani, C., Romano-Díaz, E., \& Ludlow, A. D. 2015, MNRAS, 446, 3330

Tornatore, L., Ferrara, A., \& Schneider, R. 2007, MNRAS, 382, 945

Truelove, J. K., Klein, R. I., McKee, C. F., et al. 1997, ApJ, 489, $\mathrm{L} 179+$

Turk, M. J., Abel, T., \& O'Shea, B. 2009, Science, 325, 601

Turk, M. J., Smith, B. D., Oishi, J. S., et al. 2011, ApJS, 192, 9

Umeda, H., \& Nomoto, K. 2003, Nature, 422, 871

Volonteri, M. 2010, A\&A Rev., 18, 279

Webster, D., Frebel, A., \& Bland-Hawthorn, J. 2016, ApJ, 818, 80

Wheeler, C., Onorbe, J., Bullock, J. S., et al. 2015, ArXiv e-prints, arXiv:1504.02466

Willman, B., \& Strader, J. 2012, AJ, 144, 76

Wise, J. H., \& Abel, T. 2007, ApJ, 671, 1559

Wise, J. H., Abel, T., Turk, M. J., Norman, M. L., \& Smith, B. D. 2012a, MNRAS, 427, 311

Wise, J. H., Demchenko, V. G., Halicek, M. T., et al. 2014, ArXiv:1403.6123, arXiv:1403.6123

Wise, J. H., Turk, M. J., Norman, M. L., \& Abel, T. 2012b, ApJ, 745,50

-. 2012c, ApJ, 745, 50

Yoshida, N., Bromm, V., \& Hernquist, L. 2004, ApJ, 605, 579

Zackrisson, E., Rydberg, C.-E., Schaerer, D., Östlin, G., \& Tuli, M. 2011, ApJ, 740, 13 